\title{
Role of mTOR in podocyte function and diabetic nephropathy in humans and mice
}

\author{
Markus Gödel,1 Björn Hartleben,, ${ }^{1}$ Nadja Herbach, ${ }^{2}$ Shuya Liu, ${ }^{1,3}$ Stefan Zschiedrich,1
} Shun Lu, ${ }^{1}$ Andrea Debreczeni-Mór, ${ }^{1}$ Maja T. Lindenmeyer, ${ }^{4}$ Maria-Pia Rastaldi, ${ }^{5}$

Götz Hartleben, ${ }^{6}$ Thorsten Wiech, 7 Alessia Fornoni, ${ }^{8}$ Robert G. Nelson, ${ }^{9}$ Matthias Kretzler, ${ }^{10}$

Rüdiger Wanke, ${ }^{2}$ Hermann Pavenstädt, ${ }^{11}$ Dontscho Kerjaschki, ${ }^{12}$ Clemens D. Cohen, ${ }^{4}$

Michael N. Hall, ${ }^{13}$ Markus A. Rüegg, ${ }^{13}$ Ken Inoki, ${ }^{14}$ Gerd Walz, ${ }^{1}$ and Tobias B. Huber, ${ }^{1,3,15}$

${ }^{1}$ Renal Division, University Hospital Freiburg, Freiburg, Germany. ${ }^{2}$ Institute of Veterinary Pathology, Centre for Clinical Veterinary Medicine, Ludwig-Maximilians-University, München, Germany. ${ }^{3}$ Spemann Graduate School of Biology and Medicine, Albert-Ludwigs-University, Freiburg, Germany. ${ }^{4}$ Division of Nephrology and Institute of Physiology, University Hospital and University of Zürich, Zürich, Switzerland. ${ }^{5}$ Renal Research Laboratory, Fondazione IRCCS Ospedale Maggiore Policlinico and Fondazione D’Amico, Milan, Italy. ' Leibniz Institute for Age Research, Fritz Lipmann Institute, Jena, Germany. ${ }^{7}$ Department of Pathology, University Hospital Freiburg, Freiburg, Germany. ${ }^{8}$ Diabetes Research Institute, L. Miller School of Medicine, University of Miami, Miami, Florida, USA. ${ }^{9}$ National Institute of Diabetes and Digestive and Kidney Diseases, NIH, Phoenix, Arizona, USA. ${ }^{10}$ Internal Medicine, Division of Nephrology, University of Michigan, Ann Arbor, Michigan, USA. ${ }^{11}$ Department of Medicine D, University Hospital Münster, Münster, Germany. ${ }^{12}$ Department of Pathology, Medical University of Vienna, Wien, Austria. ${ }^{13 B i o z e n t r u m, ~ U n i v e r s i t y ~ o f ~ B a s e l, ~ B a s e l, ~ S w i t z e r l a n d . ~}$ ${ }^{14}$ Life Sciences Institute, Department of Molecular and Integrative Physiology, and Division of Nephology, Internal Medicine, University of Michigan, Ann Arbor, Michigan, USA. ${ }^{15}$ BIOSS Centre for Biological Signalling Studies, Albert-Ludwigs-University, Freiburg, Germany.

\begin{abstract}
Chronic glomerular diseases, associated with renal failure and cardiovascular morbidity, represent a major health issue. However, they remain poorly understood. Here we have reported that tightly controlled mTOR activity was crucial to maintaining glomerular podocyte function, while dysregulation of mTOR facilitated glomerular diseases. Genetic deletion of mTOR complex 1 (mTORC1) in mouse podocytes induced proteinuria and progressive glomerulosclerosis. Furthermore, simultaneous deletion of both mTORC1 and mTORC2 from mouse podocytes aggravated the glomerular lesions, revealing the importance of both mTOR complexes for podocyte homeostasis. In contrast, increased mTOR activity accompanied human diabetic nephropathy, characterized by early glomerular hypertrophy and hyperfiltration. Curtailing mTORC1 signaling in mice by genetically reducing mTORC1 copy number in podocytes prevented glomerulosclerosis and significantly ameliorated the progression of glomerular disease in diabetic nephropathy. These results demonstrate the requirement for tightly balanced $m$ TOR activity in podocyte homeostasis and suggest that mTOR inhibition can protect podocytes and prevent progressive diabetic nephropathy.
\end{abstract}

\section{Introduction}

Podocyte injury is a key determinant of diabetic nephropathy and glomerulosclerosis, the leading causes of chronic kidney disease in patients starting renal replacement therapy (1). However, a comprehensive molecular pathogenetic model for diabetic nephropathy and progressive glomerulosclerosis has not been established, hampering the development of effective therapeutic approaches that prevent the progression to end-stage renal disease (ESRD). The mammalian target of rapamycin (mTOR) signaling cascade controls cellular growth, survival, and metabolism. The serine/ threonine kinase mTOR is the catalytical subunit of 2 distinct complexes, mTOR complexes 1 and 2 (mTORC1 and mTORC2), that can be distinguished by their unique composition and different substrates. mTORC1 with its essential components mTOR, $\mathrm{mLST} 8$, and rapamycin-sensitive adaptor protein of $\mathrm{mTOR}$ (Raptor) promotes protein synthesis and an increase in cell size (2). The activation of $\mathrm{mTORC} 1$ predominantly results in the phosphorylation of 2 downstream targets, the ribosomal S6Kinase (S6K) and the eukaryotic translation initiation factor 4E-binding protein (4E-BP), which stimulate ribosome biogenesis and protein transla-

Authorship note: Markus Gödel and Björn Hartleben contributed equally to this work.

Conflict of interest: The authors have declared that no conflict of interest exists. Citation for this article: J Clin Invest. 2011;121(6):2197-2209. doi:10.1172/JCI44774. tion to increase cell mass $(3,4)$. Drosophila mutants for TOR and S6K exhibit a significantly reduced body size while cell numbers are unaltered $(5,6)$. The essential core of the rapamycin-insensitive complex (mTORC2) comprises mTOR, mSIN1, mLST8, and the rapamycin-insensitive subunit Rictor; mTORC2 controls cell survival and cytoskeletal organization (2). mTORC2 phosphorylates AKT at a critical site (7). In addition, mTORC2 phosphorylates conventional and atypical forms of protein kinase C (7). mTOR signaling has recently been implicated in inflammatory, metabolic, degenerative, and proliferative human diseases $(2,8)$. However, the function of mTOR in the glomerulus remains elusive and the current data are controversial (9): while some studies suggested that mTOR inhibition by rapamycin might delay or reverse glomerulopathies (10-14), other studies documented an increase in proteinuria and glomerulosclerosis in patients and animal models following rapamycin treatment $(9,15-17)$. In addition, most studies so far have been based on pharmacological inhibition of mTORC1 by rapamycin. Since rapamycin affects resident as well as infiltrating cells in the kidney, this approach does not allow distinguishing the specific role of blocking mTOR in the different cell types. Furthermore, offtarget effects have been described with long-standing application of rapamycin, most notably the inhibition of mTORC2 (18). Therefore, tissue-specific analysis of mTOR signaling is required for an in-depth understanding of the functional and cell autonomous role of mTOR in diabetic nephropathy and other glomerular diseases. 
A
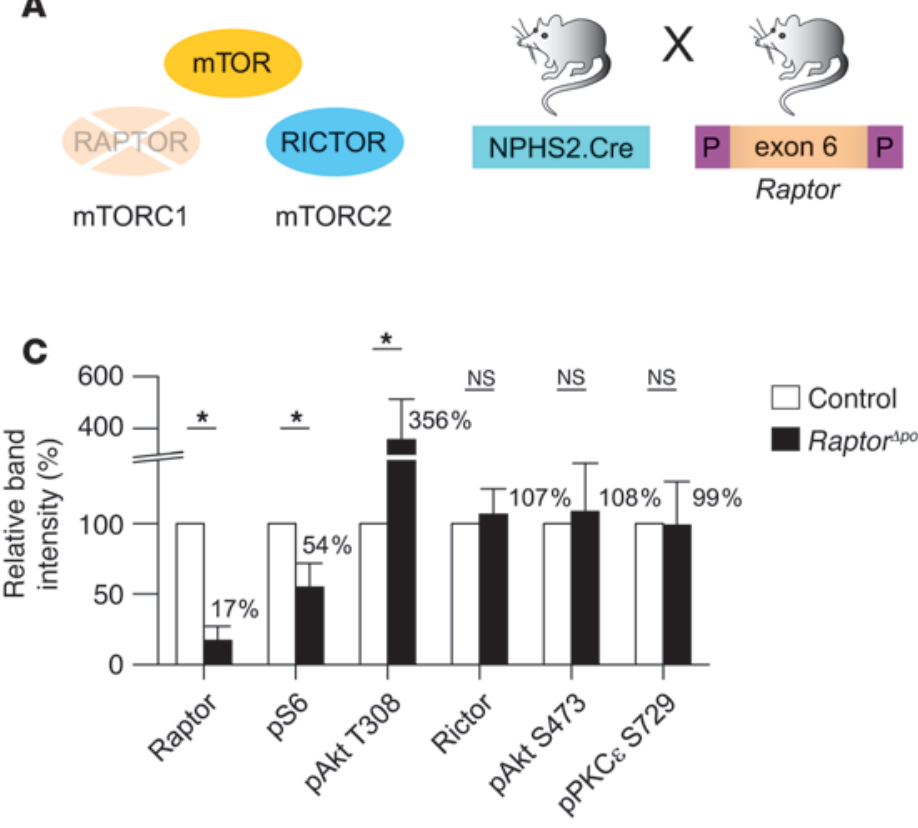

$\square$ Control
B

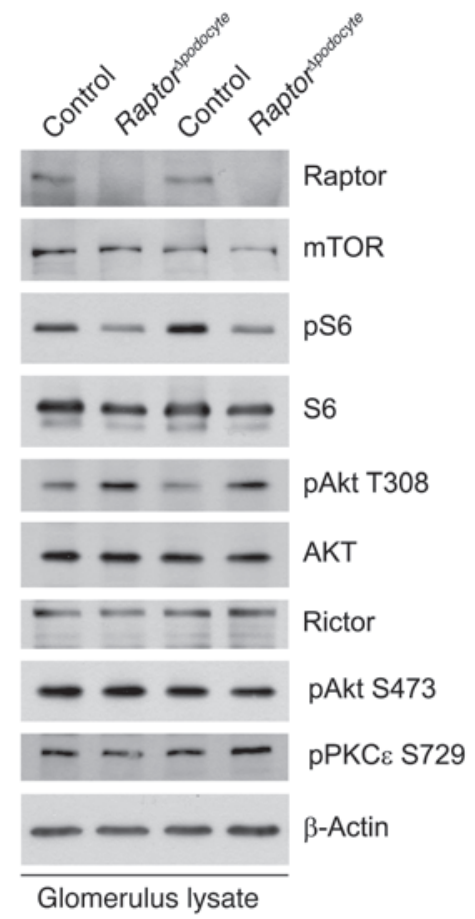

$\mathbf{F}$
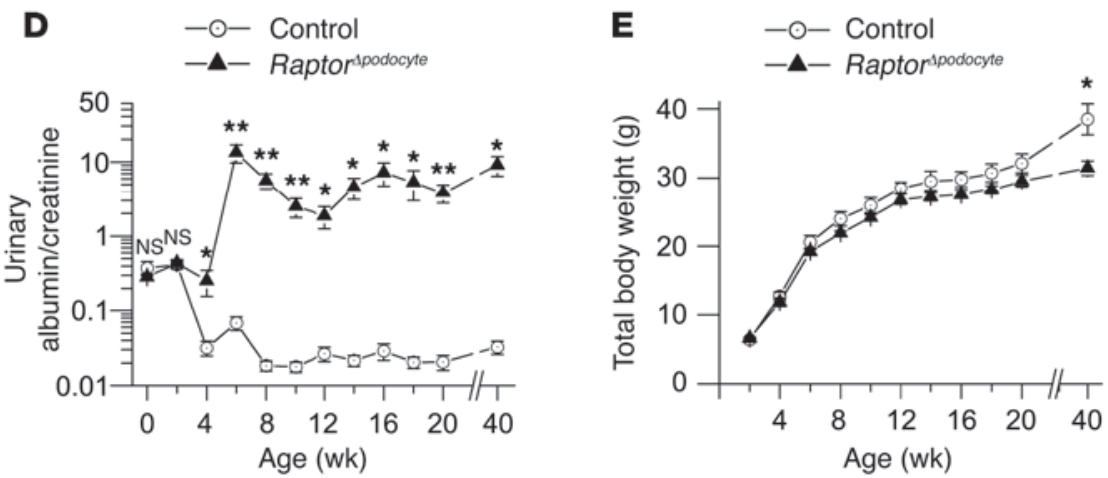

Raptoripodocyte

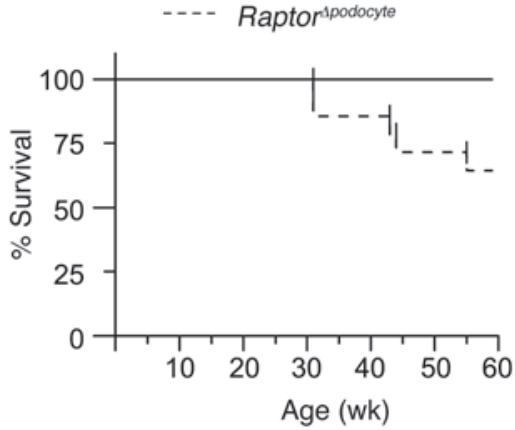

Figure 1

The mTORC1 complex is required for glomerular function. (A) Schematic illustration of the generation of podocyte-specific Raptor-deficient (Raptorspodocyte) mice to interrupt mTORC1 signaling. (B) Western blot analysis of isolated glomeruli from Raptorspodocyte and control littermates. (C) Densitometric analysis confirmed significant reduction of glomerular Raptor and pS6 levels and an upregulation of pAkt T308 ( $n=3$ each; $\left.{ }^{*} P<0.05\right)$. (D) 40-week follow-up of Raptor ${ }^{\Delta p o d o c y t e}$ mice for proteinuria and (E) body weight $\left(n=9\right.$ control, $n=10$ Raptor ${ }^{\Delta p o d o c y t e}$ male mice; ${ }^{*} P<0.05$; $\left.{ }^{* \star} P<0.01\right)$. (F) Raptor ${ }^{\Delta p o d o c y t e}$ mice displayed an increased mortality after 40 weeks of age. Data are expressed as the mean $\pm \mathrm{SEM}$.

Here, we present a comprehensive genetic analysis of mTOR-associated regulatory events to reveal the fundamental role of this pathway in glomerular development, maintenance, and disease.

\section{Results}

Podocyte specific loss of $m$ TORC1 causes proteinuria and progressive glomerulosclerosis. The clinical hallmark of podocyte injury is proteinuria, which has been documented under various acquired conditions including treatment with the mTORC1 inhibitor rapamycin $(9,15-17)$. To define the podocyte intrinsic role of mTORC1 in a model system, we generated podocyte-specific mTORC1 knockout mice (Raptorspodocyte) by crossing Raptor-floxed mice (Raptorflox/flox) with a NPHS2.Cre deleter strain (refs. 19-21 and Figure 1A). Next, we biochemically analyzed the mTOR signaling cascade in Raptor ${ }^{\text {Apodocyte }}$ mice. Lysates from purified glomeruli of Raptor ${ }^{\Delta p o d o c y t e}$ mice and control littermates were compared. Although podocytes account only for about $30 \%$ of all glomerular cells, podocyte-specific deletion resulted in a remarkable reduction of glomerular raptor protein in Raptorspodocyte mice, whereas the total protein levels of mTOR remained unchanged (Figure 1, $\mathrm{B}$ and $\mathrm{C}$ ). In agreement with the glomerular deletion of Raptor, the phosphorylation of the mTORC1 downstream target S6 was significantly decreased, by about 50\% (Figure 1, B and C). In addition, phosphorylation of Akt on residue Thr308 was strongly increased in Raptorspodocyte mice (Figure 1, B and C). Activation of S6K by mTORC1 causes feedback inhibition of the insulin/IGF1 pathway by affecting the levels and the phosphorylation of IRS-1 $(22,23)$. Thus, activation of Akt on residue Thr308 in Raptor-deficient podocytes is probably due to the failure to activate S6K and to prevent phosphorylation of IRS-1. The glomerular protein levels of Rictor as well as the phosphorylation of downstream targets of mTORC2 such as PKCE and Akt 
A

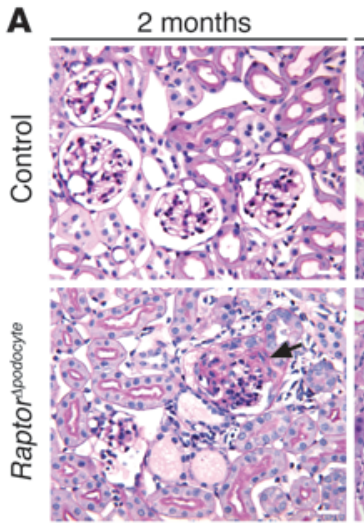

C Control

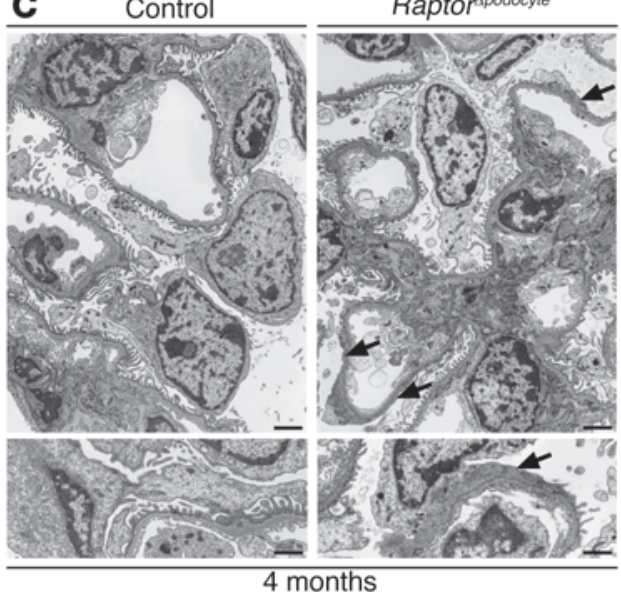

E

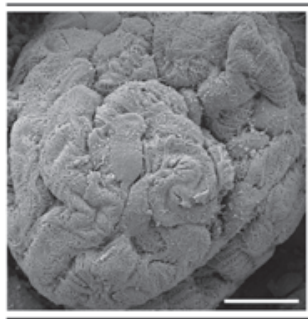

10 months

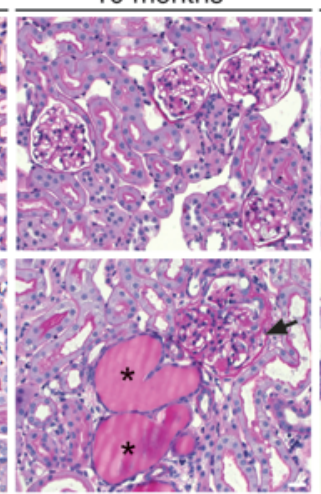

Control

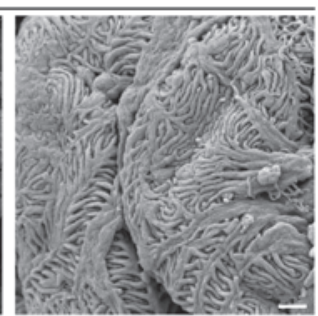

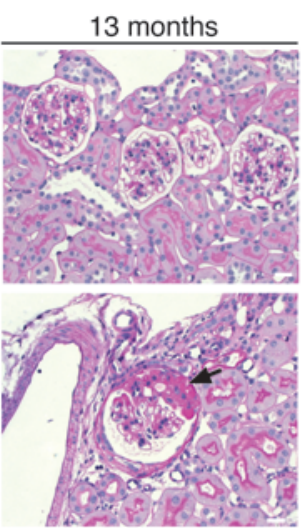

D Control

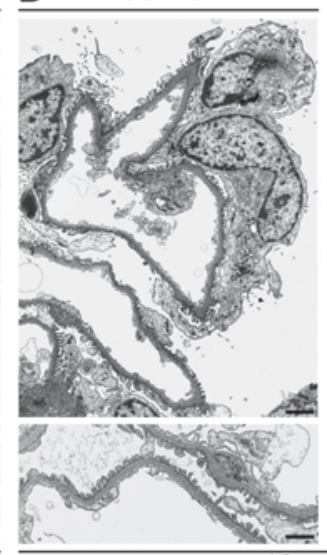

B


Raptor ${ }^{\text {spodocye }}$
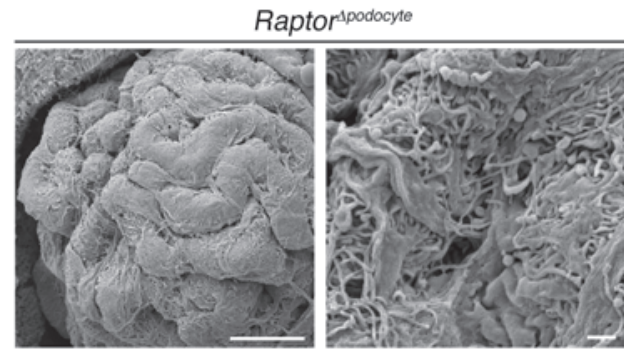

12 months

\section{Figure 2}

Podocyte-specific deletion of the mTORC1 complex results in progressive glomerulosclerosis. (A and B) Raptorspodocyte mice developed progressive glomerulosclerosis between 2 and 12 months of age. Asterisks in photographs indicate proteinaceous casts in dilated tubules. Arrows indicate glomerulosclerosis with synechia formation $\left(n=4\right.$ mice each; $\left.{ }^{*} P<0.05\right)$. Data are expressed as the mean \pm SEM. (C and $\left.\mathbf{D}\right)$ TEM analyses identified foot process effacement (arrows) at 4 and 12 months of age. (E) SEM analysis of foot processes at 12 months of age. Scale bars: $20 \mu \mathrm{m}$ (A); $2 \mu \mathrm{m}$ (C and D, upper panel); $1 \mu \mathrm{m}$ (C and D, lower panel); $10 \mu \mathrm{m}$ (E, first and third scanning electron micrograph); $1 \mu \mathrm{m}$ (E, second and fourth scanning electron micrograph).

S473 were not altered (Figure 1, B and C). Although these data indicate that mTORC2 is not upregulated in Raptor-deficient glomeruli, we cannot exclude that mTORC2 activity might be locally activated in the podocyte. Raptorspodocyte mice were born at the expected Mendelian ratios. At up to 2 weeks of age, Raptor ${ }^{\Delta \text { podocyte }}$ mice were indiscernible from their WT littermates regarding albuminuria levels (Figure 1D), weight (Figure 1E), and glomerular histology (Supplemental Figure 1; supplemental material available online with this article; doi:10.1172/JCI44774DS1). However, at 4 weeks of age, Raptor ${ }^{\text {spodocyte }}$ mice developed significant albuminuria, which increased at 8 weeks of age (albuminuria at 8 weeks of age: urinary albumin/creatinine: mean 23.32; urinary albumin: mean $1088.90 \mathrm{mg} / \mathrm{l}$ ) (Figure 1D). The proteinuria was associated with weight loss and increased lethality after 8 months of age (Figure 1, E and F).

In histological sections, Raptorspodocyte mice displayed progressive glomerulosclerosis and proteinaceous casts in dilated tubules; the glomerulosclerosis was most prominent in juxtamedullary glomeruli (Figure 2, A and B, and ref. 24). The immunofluorescence expression patterns of the slit diaphragm proteins Nephrin, Podocin, and Par3 in nonsclerosed glomeruli of Raptorspodocyte mice were 


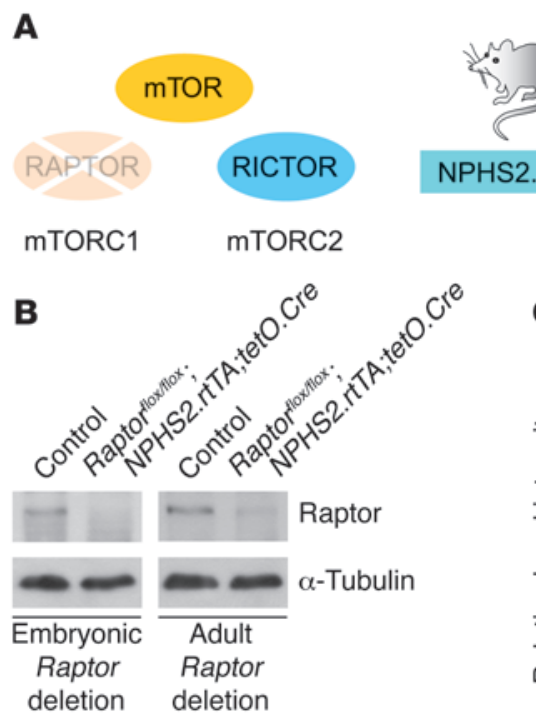

\footnotetext{
Glomerulus lysate
}
C $\square$ Control
口 Raptor ${ }^{\text {floxflox; }}$,NPHS2.rtTA;tetO.Cre

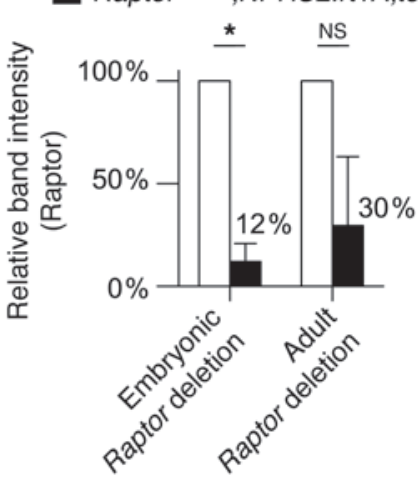

D

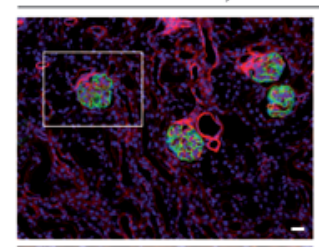

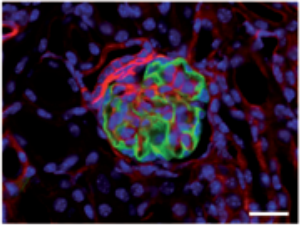

Embryonic doxycyclin induction

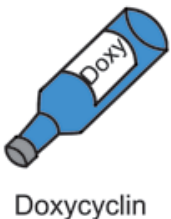

Doxycyclin


Embryonic Raptor deletion

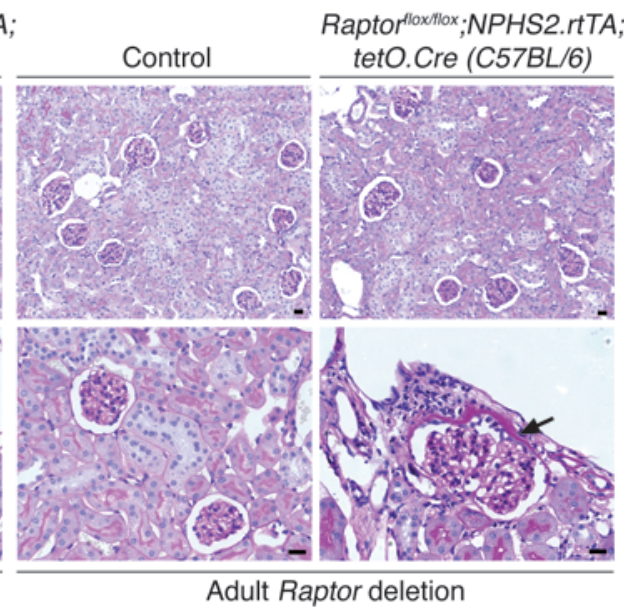




\section{Figure 3}

Time-specific deletion of Raptor indicates the importance of mTORC1 activity during glomerular development. (A) Schematic illustration of the generation of doxycycline-inducible podocyte-specific Raptor-deficient mice. (B) Western blot analysis of isolated glomeruli confirmed Raptor deletion after embryonic or adult doxycycline induction. (C) Densitometric analysis $\left(n=3\right.$ each; $\left.{ }^{*} P<0.05\right)$. (D) Embryonic and adult doxycycline induction of $m T / m G ; N P H S 2$.rtTA;tetO. Cre reporter mice resulted in podocyte-specific GFP expression. Arrow indicates incomplete GFP expression in podocytes after adult induction. (E) Urinary albumin excretion rates after embryonic or adult Raptor deletion, respectively (C57BL/6 background; embryonic Raptor deletion: $n=7$ controls, $n=20$ Raptor deletion; adult Raptor deletion 2 months after doxycycline administration, $n=13$ each, 6 months after doxycycline administration, $n=8$ controls, $n=9$ Raptor deletion; $\left.{ }^{* *} P<0.001\right)$. (F) Adult Raptor deletion on ICR background caused significant albuminuria ( $n=14$ controls, $n=19$ Raptor deletion; $\left.{ }^{* *} P<0.001\right)$. Data are expressed as the mean \pm SEM. (G) Histological analyses 6 months after podocyte-specific Raptor deletion (C57BL/6 background) documenting glomerulosclerosis in embryonic induced mice. In adult doxycycline-induced mice (C57BL/6 background), synechia, but no glomerulosclerosis, could be detected (arrows indicate sclerosed glomeruli or synechia, respectively, arrowheads indicate proteinaceous casts in distal tubules). Scale bars: $20 \mu \mathrm{m}$.

regular at 4 months, suggesting that the glomerulopathy was not primarily caused by reduction or redistribution of slit diaphragm protein expression (Supplemental Figure 2). TEM and SEM ultrastructural analyses revealed progressive podocyte foot process broadening and effacement, documented at 4 and 12 months of age (Figure 2, C-E). These data indicate that mTORC1 is required to maintain podocyte function and glomerular architecture.

Time-dependent deletion of Raptor indicates the importance of $m$ TORC1 activity during glomerular development. Growth of an organ during development can be controlled by alterations in either the number or the size of cells. The 2 mechanisms are fundamentally different and require distinct regulation, whereby $\mathrm{mTORC} 1$ is centrally involved in controlling cell size. The convergence of multiple growth factor-initiated pathways on mTORC1 likely allows its participation in multiple developmental processes, underlined by the absolute requirement for mTORC1 during early embryonic development $(25,26)$. In agreement with this, morphometric analysis of 8-week-old Raptorspodocyte mice indicated that glomeruli and podocytes appear to be smaller in Raptorspodocyte mice than in WT controls (Supplemental Figure 3). To determine whether podocytes might be particularly sensitive to the loss of mTORC1 activity during glomerular development, we used a conditional expression model (Tet-On system) in which the target gene is deleted only in the presence of a tetracycline derivative. In this system, the reverse tetracycline-controlled transcriptional activator $(r t T A)$ is placed under the control of the NPHS2 promoter (27). A second transgene uses the tet $O$ promoter elements upstream of a minimal CMV promoter to drive expression of Cre recombinase. We used this strategy to delete the Raptor gene either during glomerular devel-

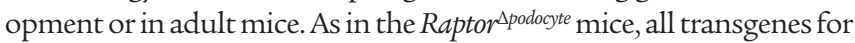
this experiment were transferred to a pure C57BL/6 strain (Figure 3A). Doxycycline was added to the drinking water of pregnant female animals and continued throughout gestation and nursing to initiate Cre-mediated excision of Raptor in developing glomeruli. To initiate Cre-mediated excision of Raptor in mature glomeruli, doxycycline was added to the drinking water of 8-week-old mice. Western blot analysis confirmed the reduction of glomerular raptor protein levels in mice induced in utero or after 8 weeks of age (Figure 3, B and C). We further assessed the efficiency of doxycycline-regulated Cre expression by crossing the double-transgenic NPHS2.rtTA;tetO.Cre mice to the $m T / m G$ transgenic reporter strain, which carries a loxP-flanked Tomato cassette (28). Upon Cre-mediated excision of membranetargeted tandem dimer Tomato $(\mathrm{mT})$, an alternate reporter protein, membrane-targeted GFP ( $\mathrm{mG})$, is expressed. Immunofluorescence of kidney sections indicated that almost all podocytes were positive for the excision event in early-induced mice (Figure 3D), whereas most, but not all (ca. 80\%) podocytes were positive for the excision event in the late-induced (8 weeks) group. This finding is in agreement with published work documenting a high but not complete excision rate of target genes in adult mice using the Tet-On system (29), though the Tet-On system-induced gene deletion in these cases caused severe glomerular defects in adult mice $(27,29)$. As expected, the inducible strategy recapitulated the phenotype observed after conventional Cre excision when doxycycline was administered to pregnant female animals to initiate early Cre expression in Raptorflox/flox;NPHS2.rtTA;tetO. Cre mice (Figure 3E). However, when Raptor deletion was induced in adult mice, the levels of protein excretion were higher compared with controls, but did not reach statistical significance (albumin/ creatinine ratios 2 months after doxycycline induction: induced Raptorflox/flox; NPHS2.rtTA; tetO.Cre: mean $=0.23 \pm 0.07$; control littermates mean $=0.13 \pm 0.06 ; n=13$ each) (Figure 3E). Histological alteration in the doxycycline-treated Raptorfloxfflox;NPHS2.rtTA;tetO.Cre embryonic C57BL/6 mice resembled those of the Raptorflox/flox;NPHS2.Cre animals, whereas histology of the late-induced (8 weeks, C57BL/6 background) did not depict any glomerulosclerosis, although some milder glomerular lesions such as glomerular synechia could be observed (Figure 3G). In context of the high interindividual variability of proteinuria in patients receiving $\mathrm{mTOR}$ inhibitors, we speculated that the genetic background as modifying factor might contribute to the maintenance phenotype of Raptor deficiency in adult mice. To prove this hypothesis, we transferred Raptorflox/flox;NPHS2.rtTA;tetO. Cre mice to an ICR background (IcrTac:ICR; Taconic USA), which is known to be more sensitive toward glomerular diseases than that of C57BL/6 mice. Strikingly, in these mice, Raptor deletion at 8 weeks of life induced significant proteinuria (Figure $3 \mathrm{~F}$ ). Taken together, these data indicate that mTORC1 is especially important for podocyte development and growth. Additionally mTORC1 is required for glomerular maintenance under homeostatic conditions, whereby this effect is modified by the genetic background of mice.

Synergistic action of $m$ TORC1 and $m$ TORC2 complexes are required for glomerular homeostasis. MTORC1 and MTORC2 phosphorylate different substrates to regulate distinct cellular functions. In contrast to mTORC1, mTORC2 is largely rapamycin-insensitive and phosphorylates cellular targets such as AKT, SGK1, and PKC to control cell survival and cytoskeletal organization (2). However, these effects vary among different cell types and the function of mTORC2 in the glomerulus has not been defined. In podocyte-specific mTORC2-deficient mice (Rictor ${ }^{\Delta \text { podocyte }}$ ) (Figure 4A), the level of Rictor and the activation state of PKCE, which is a well-characterized substrate of mTORC2 (30), was significantly reduced (Figure 4B). Rictor ${ }^{\Delta \text { podocyte }}$ mice did not display any obvious clinical (Figure 4C), histological (Figure 4D), or ultrastructural abnormalities (Figure 4, E and F) during a 2-year follow-up. However, Rictor ${ }^{\Delta p o d o c y t e}$ mice exposed to stress such as BSA overload $(31,32)$ developed significantly higher transient albuminuria than control littermates (Figure 4G), suggesting that mTORC2 might play a role in podocyte adaptation and foot process reorganization in response to stress. 

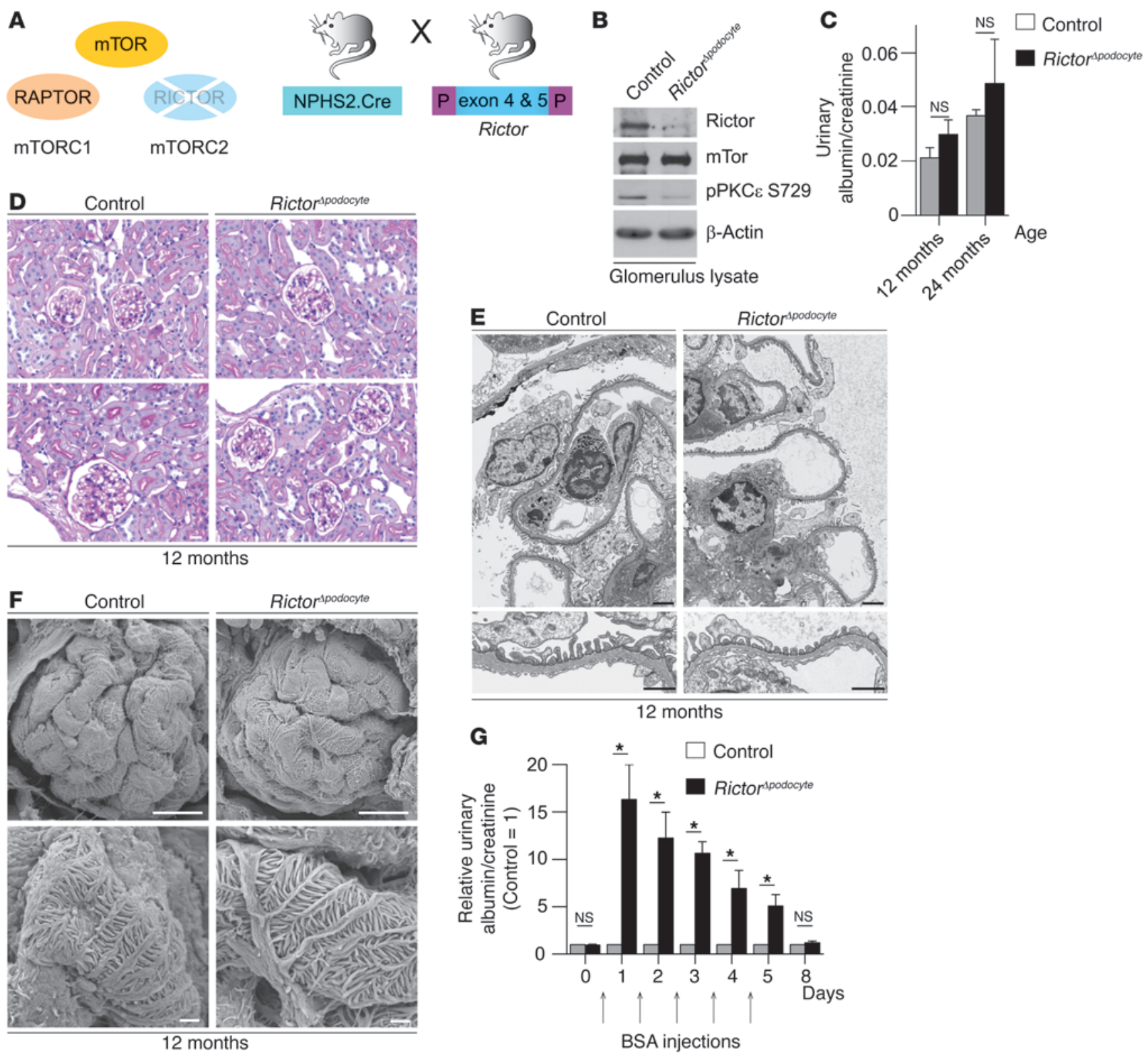

\section{Figure 4}

Podocyte-specific knockout of the mTORC2 complex results in reduced ability to adapt to stress. (A) Schematic illustration of the generation of podocyte-specific Rictor-deficient mice (Rictorspodocyte) to interrupt mTORC2 signaling. (B) Western blot analysis of isolated glomeruli from Rictor $\triangle$ podocyte mice confirmed the significant reduction of Rictor and $\mathrm{pPKC} \varepsilon \mathrm{S} 729$. (C) Rictorspodocyte mice displayed no significant increase in albuminuria at 12 and 24 months of age ( $n=6$ control and $n=10$ Rictorspodocyte mice; 12 months, $P=0.11 ; 24$ months, $P=0.24)$. (D-F) No obvious histological and ultrastructural lesions in 12-month-old Rictorspodocyte mice. (G) Rictor ${ }^{\Delta p o d o c y t e}$ mice exhibited a significantly increased transient albuminuria in the BSA overload model compared with control mice $\left(n=8\right.$ control and $n=8$ Rictor ${ }^{\Delta p o d o c y t e}$ mice; $\left.{ }^{*} P<0.05\right)$. Scale bars: 20 um (D); $2 \mu \mathrm{m}$ (E, upper panel); $1 \mu \mathrm{m}$ (E, lower panel); $10 \mu \mathrm{m}$ (F, upper panel); $1 \mu \mathrm{m}$ (F, lower panel). Data are expressed as the mean \pm SEM.

Strikingly, the combined podocyte-specific deletion of mTORC1 and mTORC2 (Raptor/Rictor ${ }_{\text {Lpodocyte }}$ (Figure 5A) caused a pronounced kidney phenotype with massive proteinuria (Figure 5B) and growth retardation (Figure 5, C and D). The severe glomerulosclerosis (Figure 5E) presented with circumferential synechia, crescent formation, vacuolization of podocytes, and often complete glomerular obsolescence at 6 weeks of age. Massive foot process effacement was documented by electron microscopy (Figure 5F). Consistent with the dramatic phenotype causing renal failure as documented by increased serum creatinine levels (Figure 5G), most Raptor/Rictor ${ }^{\text {Ppodocyte }}$ mice died by 8 weeks of age (Figure $5 \mathrm{H}$ ). These findings reveal that both mTOR complexes are required for podocyte homeostasis and that mTORC2 plays an unexpected role in podocyte function and stress adaptation.

mTORC1 activation is a molecular signature of diabetic nephropathy. Recent studies suggest that the mTOR pathway plays an important role in mechanisms underlying the progression of glomerular diseases (10-14). Many glomerular diseases such as dia- 
A

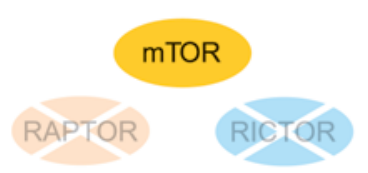

mTORC1 mTORC2
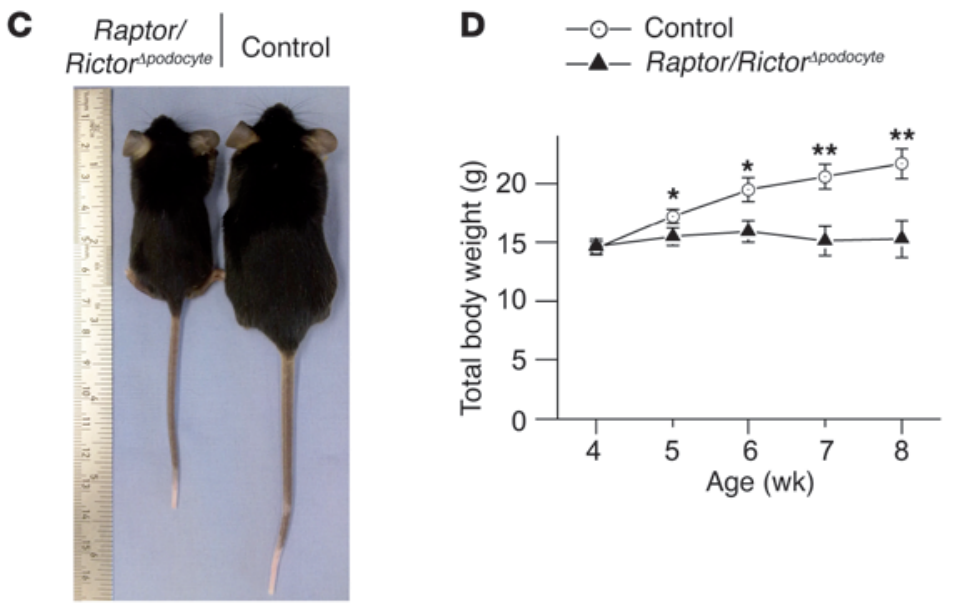

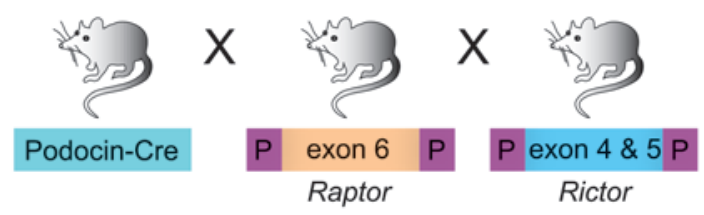

B

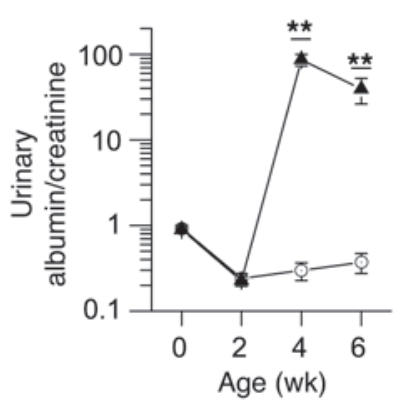

$\underline{\mathbf{F}}$

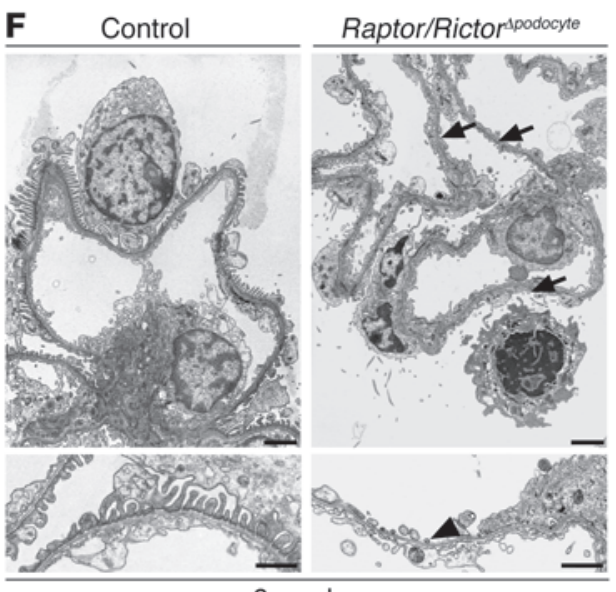

G

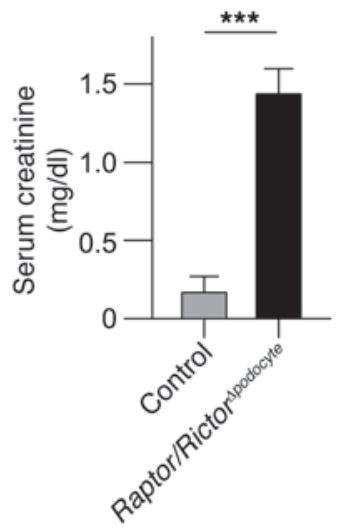

E

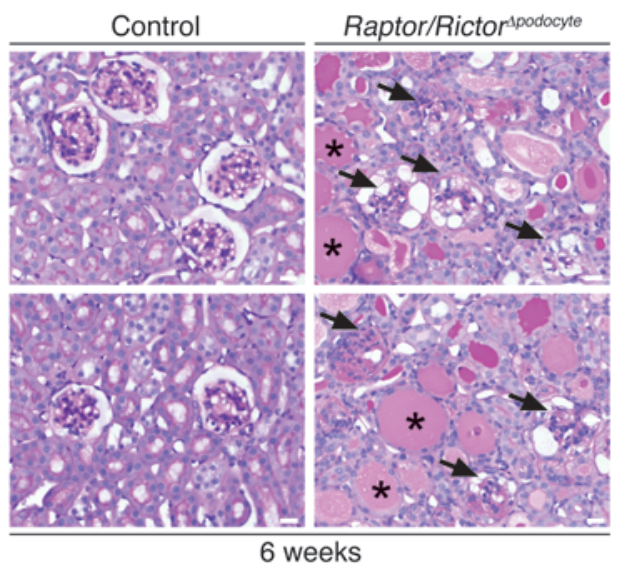

6 weeks

H $\quad$ Control -.-- Raptor/Rictor ${ }^{\text {pododecye }}$

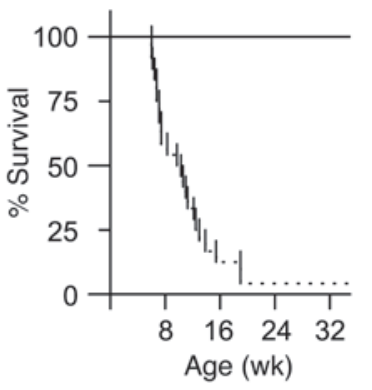

\section{Figure 5}

Synergistic action of mTORC1 and mTORC2 complexes are required for glomerular homeostasis. (A) Schematic illustration of the generation of podocyte-specific Raptor- and Rictor-deficient mice (Raptor/Rictorspodocyte) to interrupt mTORC1 and mTORC2 signaling. (B) Raptor/Rictor ${ }^{\Delta p o d o c y t e}$ developed an early onset massive albuminuria $\left(n=7\right.$ control and $n=6$ Raptor/Rictor ${ }^{\Delta p o d o c y t e}$ mice; $\left.{ }^{*} P<0.01\right)$. (C and D) Raptor/Rictor $\Delta$ podocyte mice exhibited significant growth retardation after 5 weeks of age $(n=8$ control and $n=6$ Raptor/Rictor $\Delta$ podocyte mice; $\left.{ }^{\star} P<0.05,{ }^{* *} P<0.01\right)$. (E) Histological analyses displayed glomerulosclerotic changes with circumferential synechia, crescent formation, vacuolization of podocytes, sometimes complete glomerular obsolescence, and proteinaceous casts in dilated distal tubules. Arrows indicate sclerotic glomeruli; asterisks indicate proteinaceous casts. (F) Raptor/Rictorspodocyte mice showed global foot process effacement or loss of foot processes with denudation of the basement membrane in ultrastructural analyses (arrows depict foot process effacement; arrowhead indicates loss of foot processes). (G) Raptor/Rictor ${ }^{\Delta p o d o c y t e}$ mice developed renal failure with increased serum creatinine ( $n=9$ control and $n=5$ Raptor/Rictor ${ }^{\Delta p o d o c y t e}$ mice; $\left.{ }^{* \star} P<0.0001\right)$ and $(\mathbf{H})$ died between 6 and 12 weeks of age. Scale bars: $20 \mu \mathrm{m}(\mathbf{E}) ; 2 \mu \mathrm{m}(\mathbf{F}$, upper panel); $1 \mu \mathrm{m}(\mathbf{F}$, lower panel). Data are expressed as the mean \pm SEM. 
A
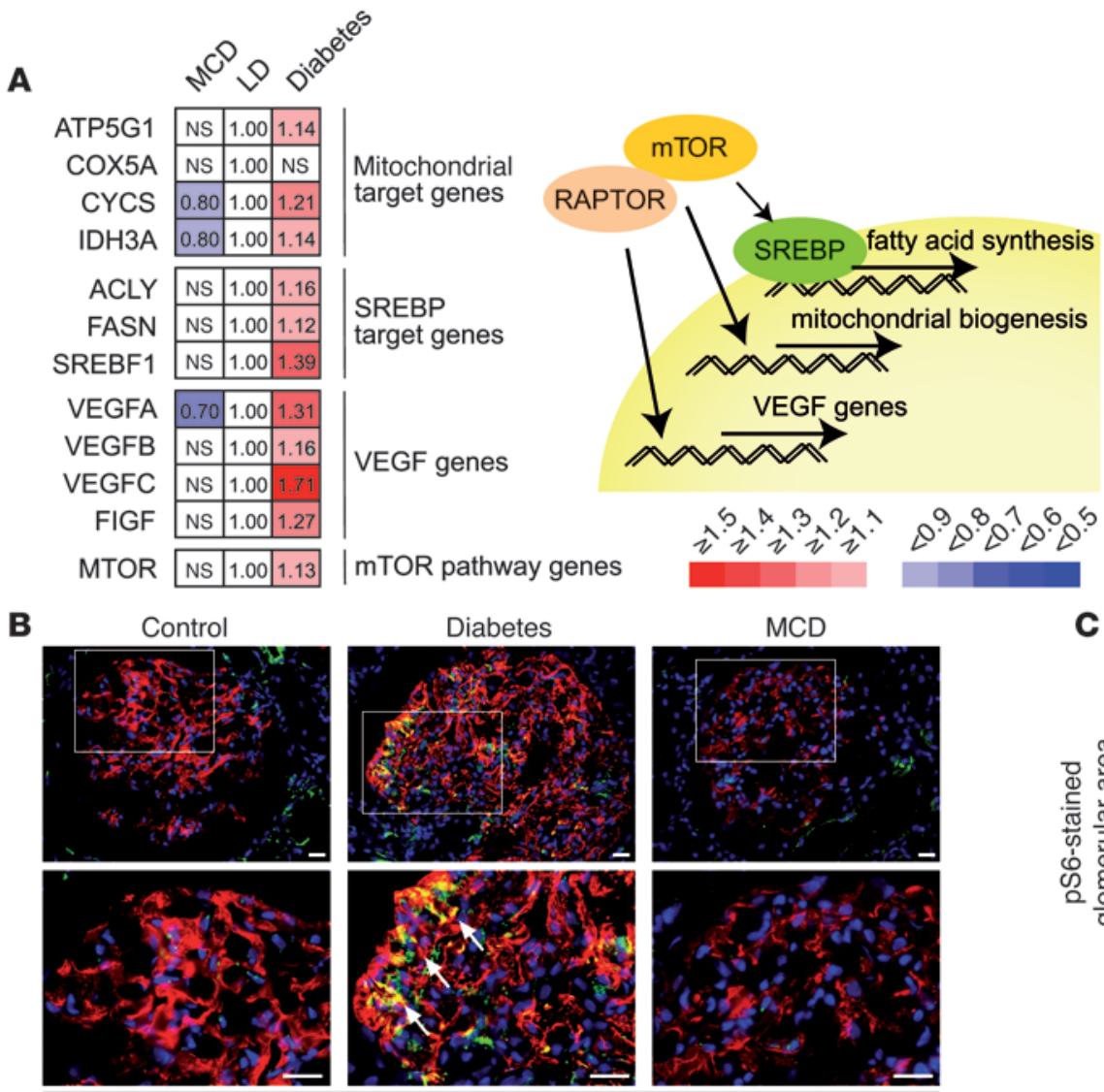

pS6/Synaptopodin
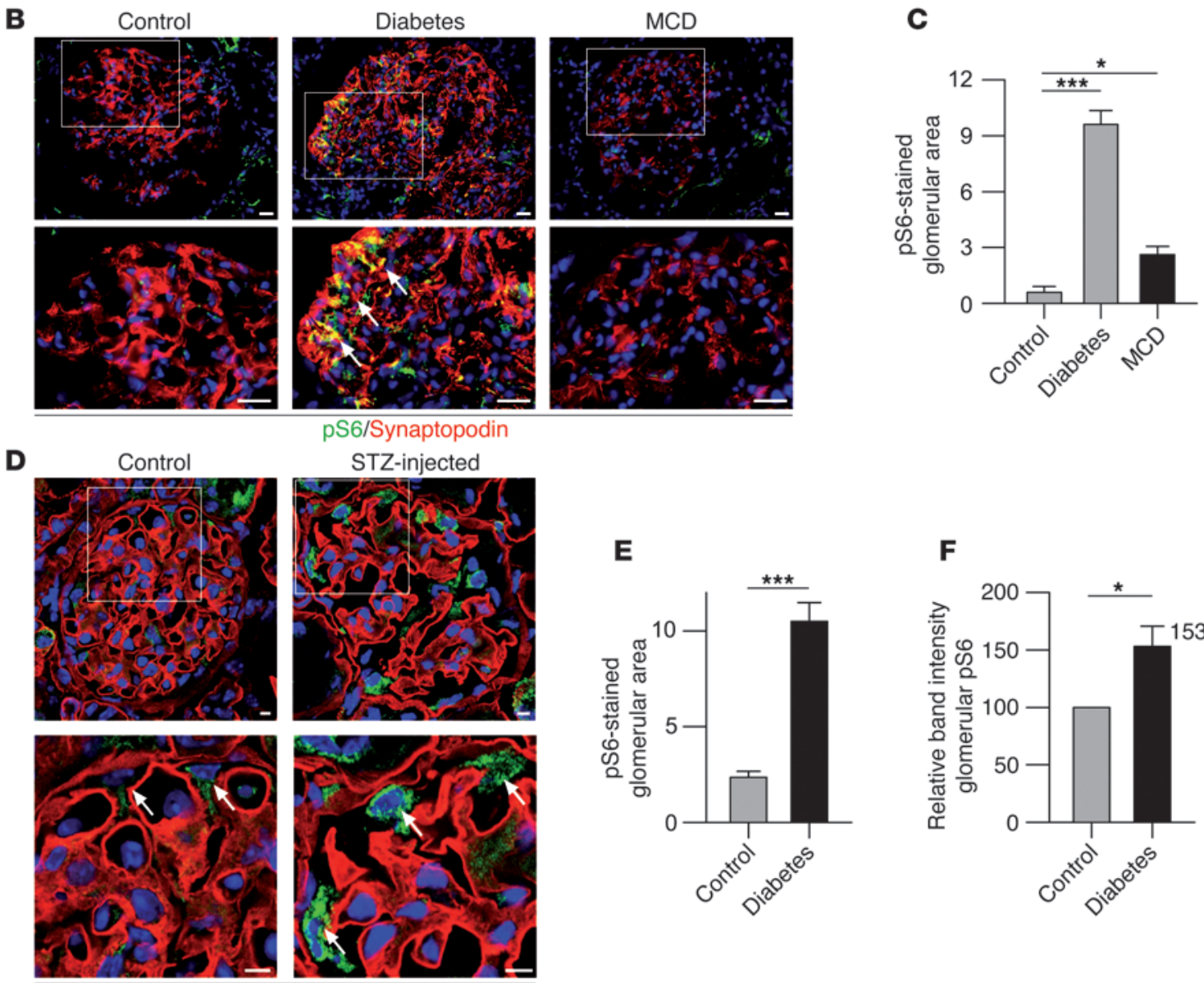

pS6/Nidogen

\section{Figure 6}

mTORC1 hyperactivation is a molecular signature of diabetic nephropathy. (A) Glomerular gene expression data of microdissected glomeruli from patients with glomerulopathies; very early diabetic nephropathy (diabetes; $n=22$ ), $\operatorname{MCD}(n=5)$, and controls (pretransplant allograft biopsies, LD, $n=18$ ). mTOR/Raptor target gene expression was upregulated in diabetic nephropathy, but not in MCD. (B) Upregulation of pS6 in glomeruli of patients with diabetic nephropathy, but not in patients with MCD (arrows indicate pS6 signal). (C) Quantitative analysis of glomerular pS6-stained area in glomeruli of patients ( $n=3$ control, $n=5$ for diabetes and minimal change; ${ }^{\star} P<0.05$, $\left.{ }^{* \star \star} P<0.0001\right)$. (D) Upregulation of pS6 in podocytes of STZ-induced diabetic mice (arrows indicate podocytes). (E) Quantitative analysis of glomerular pS6-stained area in glomeruli of diabetic mice ( $n=3$ mice each). (F) Densitometric analysis after Western blotting of pS6 levels in glomerular lysates of control and STZ-injected diabetic mice ( $n=4$ mice each). Scale bars: $20 \mu \mathrm{m}(\mathbf{B}) ; 5 \mu \mathrm{m}$ (D). Data are expressed as the mean \pm SEM. 
betic nephropathy are preceded by glomerular hypertrophy and enlargement of podocytes $(33,34)$. Since mTOR controls cell size (2), these findings suggest an involvement of mTOR; however, conclusive evidence demonstrating elevated mTORC1 activity in podocytes of diabetic models is lacking. To test the hypothesis that mTOR activation is associated with chronic glomerular diseases, we took advantage of the fact that mTORC1 directly phosphorylates and activates transcription factors, thereby directly increasing mRNA levels of some well-described gene targets such as the SREBP, VEGF, and mitochondrial target genes. We first analyzed transcriptional targets of mTOR in microdissected glomeruli from patients with diabetic nephropathy (35). To circumvent the detection of rather unspecific expression signals at late stages of diabetic nephropathy potentially reflecting changes due to glomerular scarring, we analyzed the glomerular gene expression profile from Pima Indians at very early stages of diabetic nephropathy $(n=22)$ and compared the data to nonprogressive proteinuric states such as that in minimal change disease (MCD) $(n=5)$ and healthy controls (living allograft donors [LD]; $n=18$ ). Strikingly, both mTORC1 target genes and mTOR mRNA itself were induced in progressive diseases, whereas transcript levels were unchanged or even repressed in MCD compared with controls (Figure 6A). Consistent with this finding, immunofluorescence staining of biopsy samples for phosphorylated S6 (pS6) as a marker of mTORC1 activity confirmed increased pS6 levels in podocytes of patients with diabetic nephropathy (Figure 6, B and C). Profound activation of mTORC1 and phosphorylation of S6 was also observed by immunofluorescence staining and Western blot analysis of pS6 in an animal model of diabetic nephropathy (streptozotocin [STZ] model) (Figure 6, D-F), confirming the association of mTORC1 activation and diabetic nephropathy.

Podocyte-specific genetic inbibition of $m$ TOR activation prevents progressive glomerular diseases. We next investigated whether interference with mTORC1 activation ameliorates the progression of renal disease involving the podocyte. Since complete deletion of mTORC1 in podocytes resulted in glomerular disease, we generated mice lacking only one Raptor allele in podocytes (Raptor Hetpodocyte) (Figure 7A). Western blot analysis from glomerular lysates confirmed a reduction of raptor protein and $\mathrm{pS} 6$ levels in Raptor Hetpodocyte mice (Figure 7, B and C). To induce diabetic nephropathy, mice were injected with low-dose STZ at 8 weeks of age. Blood glucose levels, blood pressure, and glomerular macrophage infiltration were similar in Raptor Het podocyte and control mice (Supplemental Figure 4). However, induction of diabetes resulted in a significant increase of mTORC1 activity, as evidenced by increased signals of pS6 in diabetic WT animals; pS6 levels were significantly lowered by deletion of 1 Raptor allele in Raptor Het podocyte mice (Figure 7, D and E). While WT diabetic mice developed substantial proteinuria by 20 weeks of age, proteinuria was significantly reduced in diabetic Raptor Het podocyte mice (Figure 7F). Strikingly, diabetic Raptor Het podocyte mice displayed significantly reduced glomerulosclerosis scores and less mesangial matrix expansion (Figure 7, G-I). Quantitative stereological analyses demonstrated that the mean podocyte volume in Raptor Het podocyte mice was reduced by about $30 \%$ compared with that in diabetic WT animals (Figure 7, $\mathrm{J}$ and $\mathrm{K}$ ). It is worth noting that similar results were also observed in animal models of type 2 diabetes (36), underlining that mTORC1 deregulation is a major driving force for glomerular diseases such as diabetic nephropathy, which can be prevented by lowering mTOR activity in podocytes.

\section{Discussion}

Loss of glomerular function is the leading cause of progressive kidney diseases resulting in ESRD. The podocyte resembles the most vulnerable component of the glomerulus, and insults that compromise the function of this specialized epithelial cell are associated with proteinuria and renal failure. The selective mTORC1 inhibitor rapamycin can cause proteinuria in both humans and animal models of kidney disease, but also has been shown to ameliorate glomerular disease, suggesting a pivotal and incompletely understood role in podocyte homeostasis. The genetic dissection of $\mathrm{mTOR}$ function provides the tools to dissect the opposing effects of mTOR activity on the integrity of the glomerular filter and the progression of podocyte disease, providing a molecular framework to correctly utilize mTOR inhibitors in the treatment of progressive glomerulopathies.

Role of $m$ TOR signaling for glomerular development and maintenance. Inhibition of mTORC1 has frequently been reported to cause proteinuria in patients (15). Rapamycin increases the risk for proteinuria in chronic allograft nephropathy (17) and is associated with increased podocyte apoptosis and development of focal segmental glomerulosclerosis (FSGS) after renal transplantation (16, 17). Utilizing podocyte-specific mTOR-deficient mice, our data document that mTOR function in podocytes is essential for the integrity of the filtration barrier. Loss of mTORC1 results in progressive glomerulosclerosis; this is further aggravated by the additional deletion of mTORC2, uncovering an unknown role for mTORC2 in podocyte homeostasis. Strikingly, the mTORC1 loss-of-function phenotype is similar to the phenotype observed in podocyte-specific insulin receptor-deficient mice (37). Together these data underline the importance of the growth hormone receptor PI3KmTORC1 axis for podocyte biology. Intriguingly, podocytes seem to be particularly sensitive to $\mathrm{mTORC} 1$ deletion during glomerular development, indicating that MTORC1 is of particular importance during podocyte growth and adaptation. mTORC1 regulates cell growth by maintaining the appropriate balance between anabolic processes, such as macromolecular synthesis and nutrient storage, and catabolic processes, such as autophagy and the utilization of energy stores. With the transition to the capillary loop state, the maturing podocytes lose their capability of cell replication. Thus, podocytes can only increase their size to compensate for further glomerular expansion during glomerular development. Podocytes need to cover the surface of the glomerular basement membrane (GBM); after the terminal differentiation of podocytes, any further increase in filtration area will require a compensatory cell growth of podocytes. Our data suggest that activation of mTORC1 allows the podocyte to participate in glomerular expansions during kidney development. In agreement with a role of MTORC1 for podocyte plasticity and growth, we observed the most pronounced glomerulosclerosis in juxtamedullary glomeruli of mTORC1-knockout mice, which represent the largest glomeruli in the kidney (38-41). In mature glomeruli, mTORC1 might be particularly important when podocytes have to adjust to environmental changes such as podocyte loss or increased mechanical pressure. In addition, by revealing the impact of different genetic mouse backgrounds on the phenotype of mTORC1 deletion in adult mice, our data point to a critical role of genetic modifiers for mTOR function. In patients receiving mTOR inhibitors, the high interindividual variability in respect to the development of proteinuria and the observation that only a very small fraction of patients develop progressive proteinuria or FSGS have been puzzling. Our animal studies indicate that genetic modifiers and environmental factors compromising podocyte function 
A
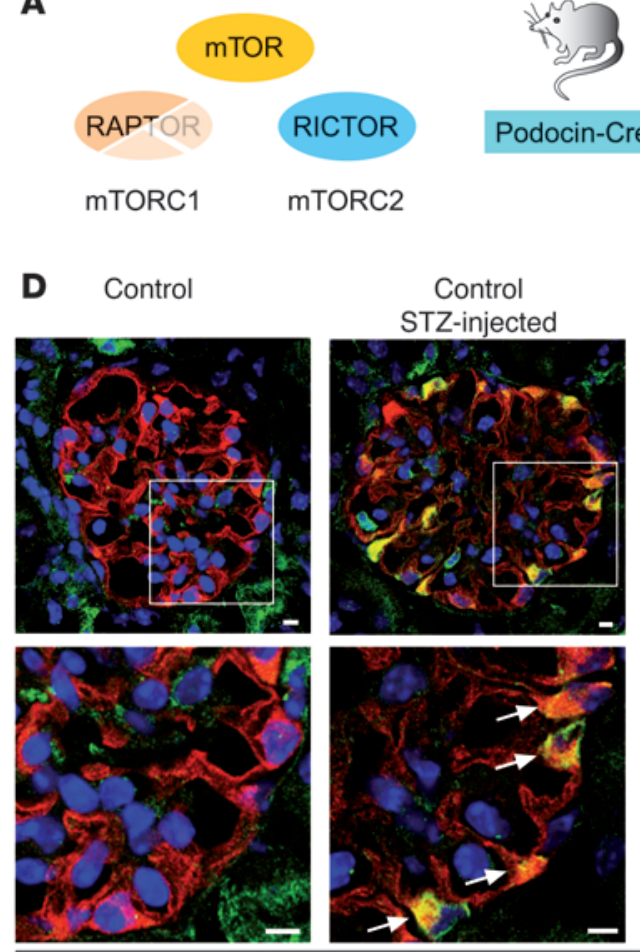

pS6/Nephrin

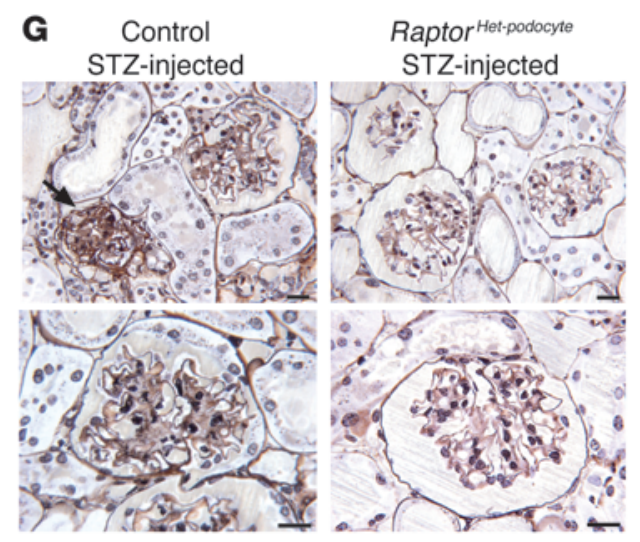

B
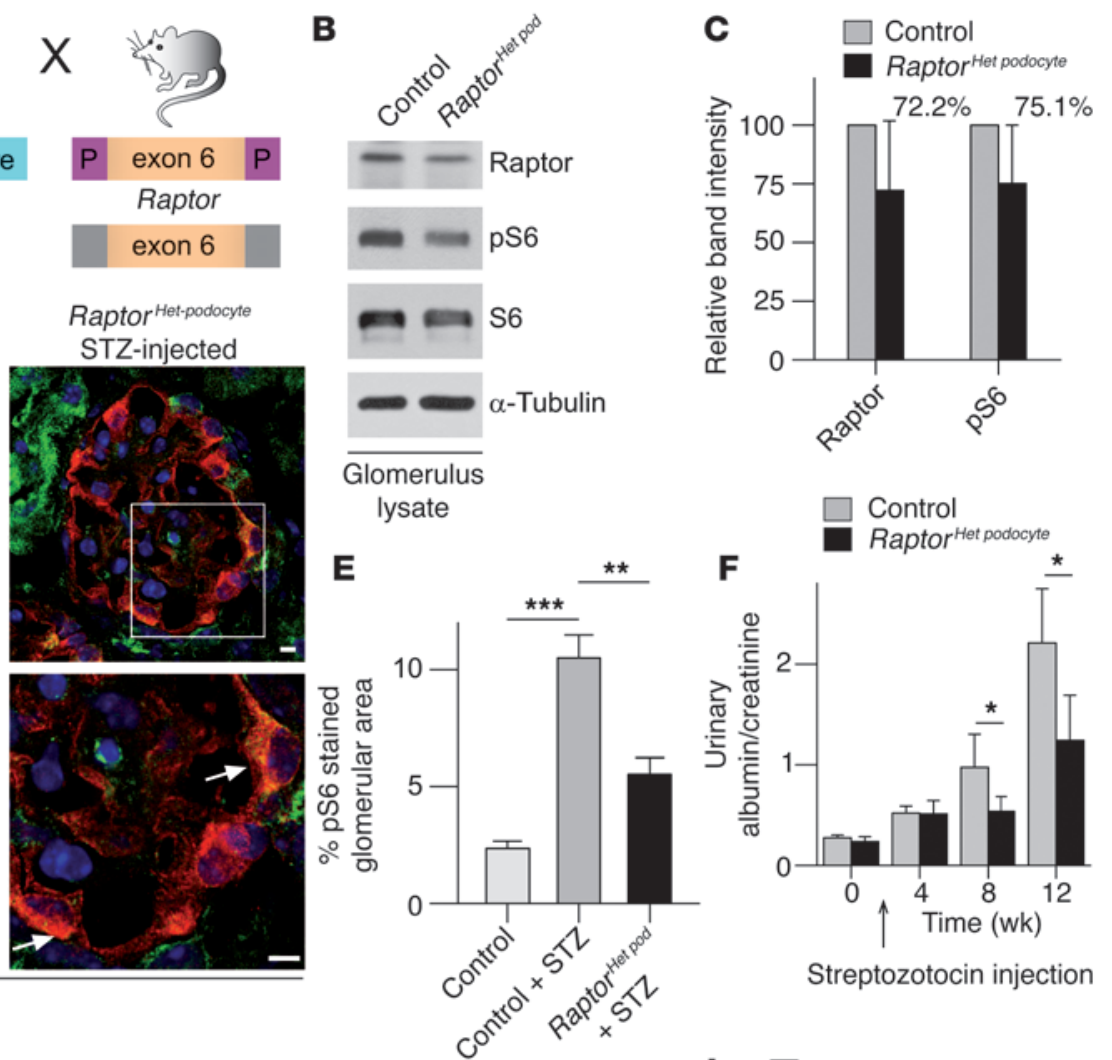

Streptozotocin injection

I $\square$ WT control $=100 \%$
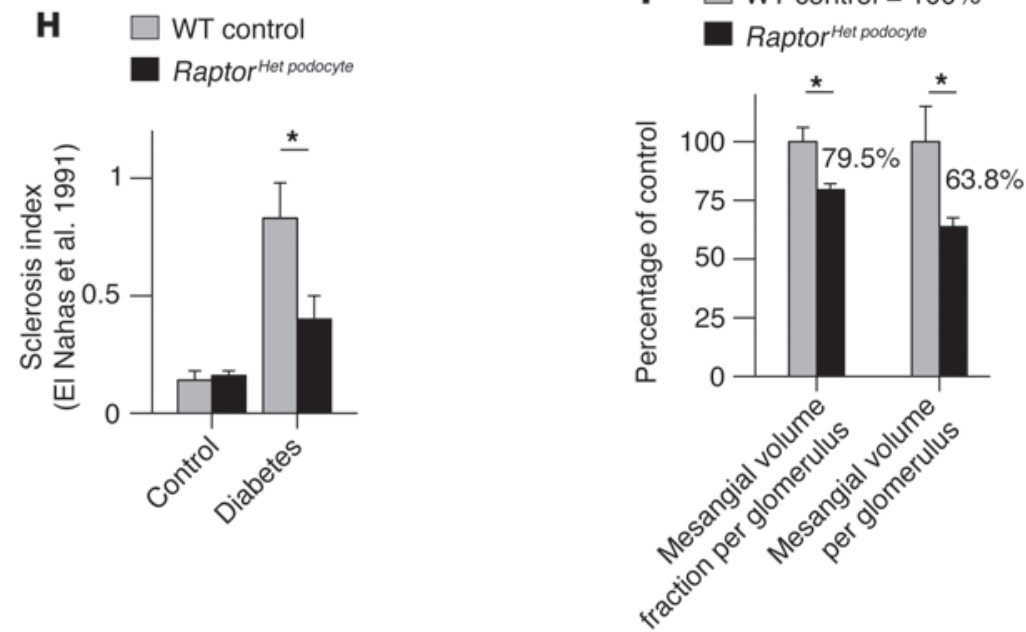

J $\square$ WT control $=100 \%$

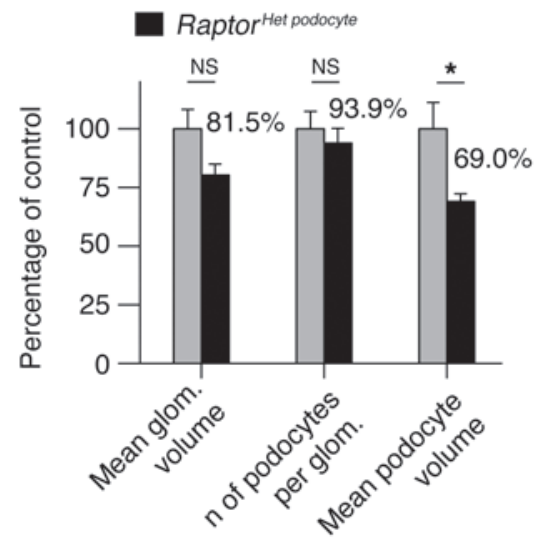

K

Control STZ-injected

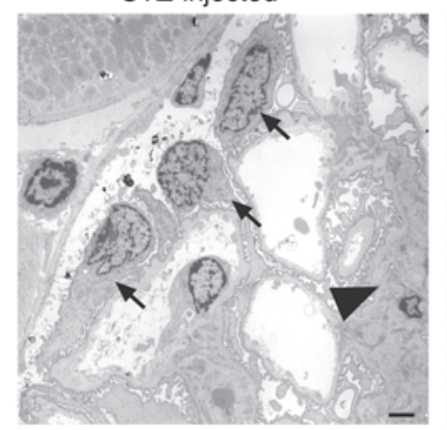

Raptor Het-podocyte STZ-injected

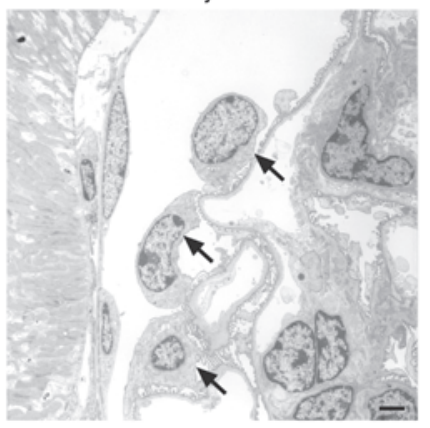




\section{Figure 7}

Podocyte-specific genetic inhibition of mTOR hyperactivation prevents progressive glomerular diseases. (A) Schematic illustration of the generation of podocyte-specific Raptor heterozygous knockout mice (Raptor Het podocyte mice) to counteract mTORC1 hyperactivation. (B) Glomerular Raptor and pS6 levels in Raptor Het podocyte mice. (C) Densitometric analysis of Raptor and pS6 levels ( $n=3$ mice). (D) Reduced S6 phosphorylation in podocytes of diabetic Raptor Het podocyte mice in the STZ model (arrows indicate pS6 signal in podocytes). (E) Quantitative analysis of glomerular pS6 stained area in glomeruli $(n=3$ mice each; $\left.{ }^{\star \star} P<0.001,{ }^{* \star} P<0.0001\right)$. (F) The Raptor Het podocyte genotype ameliorated the development of proteinuria in the STZ model ( $n=11$ control and $n=5$ Raptor Het podocyte mice; $\left.{ }^{*} P<0.05\right)$. (G) Histological analysis revealed reduced glomerulosclerosis and reduced mesangial matrix expansion in Raptor Het podocyte mice exposed to STZ (arrow indicates sclerosed glomerulus). (H) Glomerulosclerosis index (24) documenting ameliorated diabetic nephropathy in Raptor Het podocyte mice $\left(n=3\right.$ each; $\left.{ }^{*} P<0.05\right)$ (I) There was a significant increase in glomerular mean mesangial volume in WT animals compared with Raptor ${ }^{H e t}$ podocyte mice after diabetes induction, with no significant difference in mean glomerular volume $\left(n=3\right.$ each; $\left.{ }^{*} P<0.05\right)$. (J) Quantitative stereological analyses displayed a significantly increased mean podocyte volume in WT animals compared with Raptor Het podocyte mice after diabetes induction, with no significant difference in the number of podocytes per glomerulus $\left(n=3\right.$ each; $\left.{ }^{*} P<0.05\right)$. (K) Ultrastructural analysis displayed increased podocyte volume in WT animals in the STZ model (arrows indicate podocytes; arrowhead indicates mesangial matrix expansion). Scale bars: $5 \mu \mathrm{m}$ (D); $20 \mu \mathrm{m}$ (G); $5 \mu \mathrm{m}$ (K). Data are expressed as the mean \pm SEM.

are likely to predispose patients to the development of a rapamycindependent glomerulopathy precipitating the high interindividual variability of $\mathrm{mTOR}$ inhibitor-dependent proteinuria in humans.

Role of mTOR signaling in glomerular disease. Although mTOR signaling is required for podocyte development and regeneration, our data provide evidence that dysregulated activity of mTORC1 is a characteristic feature of diabetic nephropathy. Early diabetic nephropathy is characterized by hypertrophy of the glomerulus with enlargement of glomerular cells (33), especially podocytes (34). Since cell mass is essentially controlled through the mTOR pathway $(2,42)$, these findings suggest a deregulation of the mTOR pathway. Interestingly, mTORC1 activity in mature podocytes is very low under basal conditions. However, already at early stages of diabetes, we detected a significant activation of mTORC1 in mice and humans. In response to $\mathrm{mTOR}$ activation, podocytes change in a fairly stereotypical manner with cell hypertrophy, foot process effacement, and eventually detachment from the GBM. Previous reports have shown that excessive podocyte hypertrophy culminates in reduced podocyte numbers and glomerulosclerosis $(43,44)$. Although the precise molecular mechanisms of how mTORC1 deregulation might affect the cellular integrity besides podocyte hypertrophy need to be delineated, recent findings suggest that mTOR hyperactivation is associated with Notch activation, which has been shown to drive podocyte disease (45-47). In addition, autophagy is known to be negatively regulated by mTORC1 activity (7), and we have previously shown that suppression of autophagy sensitizes podocytes toward glomerular diseases, which likely contributes to podocyte injury in diabetes (48).

Another mechanistic link comes from recent insights into the role of mTOR signaling in neurons. Neurons, like podocytes, functionally depend on the specification of highly specialized cellular processes. In agreement with our findings, it was recently demon- strated that the mTOR pathway confines the polarized neuronal architecture and that mTOR hyperactivation contributes to neurological disorders (49).

In summary, our data, using defined genetically modified mouse models, demonstrate that mTOR signaling plays a major role in podocyte homeostasis. Both mTORC1 and mTORC2 are needed for podocyte development and podocyte maintenance. Glomerular disease activates mTOR in podocytes, likely in an attempt to maintain podocyte homeostasis. However, this mTOR activation, which may provide some short-term benefits, ultimately causes proteinuria and glomerulosclerosis and facilitates disease progression. Genetically reducing mTOR levels by eliminating 1 Raptor allele dramatically prevents the consequences of excessive mTOR activation, suggesting that correctly timed inhibition of mTOR activity may prevent podocyte injury and ameliorate the progression of common glomerular diseases such as diabetic nephropathy.

\section{Methods}

Mice. Mice, in which exon 6 of the Raptor gene or exons 4 and 5 of the Rictor gene, respectively, are flanked by 2 loxP sequences, have been previously reported $(20,21)$. NPHS2.Cre mice were provided by Lawrence Holzman (Renal, Electrolyte, and Hypertension Division, University of Pennsylvania School of Medicine, Philadelphia, Pennsylvania, USA) (19). Raptorfloxed mice (Raptorflox/flox) or Rictor-floxed mice (Rictorflox/flox) were crossed with NPHS2.Cre mice to generate podocyte-specific Raptor knockout mice Raptorflox/flox; NPHS2.Cre (Raptor $^{\text {Spodocyte }}$ ) or podocyte-specific Rictor-knockout mice Rictorflox/flox; NPHS2.Cre (Rictor $\left.{ }^{\Delta p o d o c y t e}\right)$ respectively. Heterozygous or NPHS2.Cre-negative litter mates served as controls. To generate podocyte-specific Raptor plus Rictor double-knockout mice (Raptor/Rictor ${ }^{\Delta p o d o c y t e}$ ), Raptorflox/flox; NPHS2.Cre were crossed with Rictorflox/flox;NPHS2.Cre mice. All Raptorflox/flox, Rictor flox/flox, and Raptor/Rictor ${ }^{\Delta p o d o c y t e}$ mice were crossed on a pure C57BL/6 background. For all STZ experiments, mice were backcrossed for 5 generations on an ICR background, which sensitizes the mice toward the development of diabetic nephropathy (IcrTac:ICRl Taconic USA).

NPHS2.rtTA;tetO.Cre mice were provided by Susan Quaggin (Samuel Lunenfeld Research Institute, Mount Sinai Hospital, University of Toronto, Toronto, Ontario, Canada) (27). To generate doxycycline-inducible podocyte-specific Raptor-knockout mice (Raptorflox/flox; NPHS2.rtTA;tetO.Cre), Raptor-floxed mice (Raptorflox/flox) were crossed with NPHS2.rtTA;tetO.Cre mice; tetO.Cre negative littermates served as control. NPHS2.rtTA;tetO.Cre mice were transferred on a pure $\mathrm{C} 57 \mathrm{BL} / 6$ background. For generation of NPHS2.rtTA;ttetO.Cre mice on an ICR background, which are more sensitive toward glomerular disease, mice were backcrossed for 5 generations (IcrTac: ICR; Taconic). For the induction of Raptor deletion, mice received doxycycline hydrochloride (Sigma-Aldrich) via drinking water $(2 \mathrm{mg} / \mathrm{ml}$ with $5 \%$ sucrose, protected from light) during pregnancy and nursing (embryonic deletion) or at 8 weeks of age (adult deletion). $m T / m G ; N P H S 2 . r t T A ; t e t O$. Cre reporter mice were generated by crossing $m T / m G$ mice (28) with NPHS2.rtTA;tetO.Cre mice and were provided by Nicola Wanner (Renal Division, University Hospital Freiburg). All animal studies were approved by the Committee on Research Animal Care, Regierungspräsidium, Freiburg.

Protein overload and subsequent analysis. Rictor ${ }^{\Delta p o d o c y t e}$ female mice $(n=8)$ received endotoxin-free BSA (Sigma-Aldrich A9430) $(250 \mathrm{mg} / \mathrm{ml}$, dissolved in PBS) intraperitoneally for 5 consecutive days $(10 \mathrm{mg} / \mathrm{g}$ body weight) $(31,32)$. Urinary albumin excretion rates were analyzed before injections and at days 1 to 8 after the first injection.

STZ-induced diabetes mellitus. Heterozygous podocyte-specific Raptorknockout mice Raptor Hetpodocyte mice $(n=5)$ and control littermates $(n=11)$ received 2 doses of intraperitoneal STZ (Sigma-Aldrich) $(125 \mu \mathrm{g} / \mathrm{g}$ body weight) in $50 \mathrm{mM}$ sodium citrate buffer on days 1 and 4 (50). Urinary 
albumin excretion rates were analyzed before injection and 4, 8, 12, and 16 weeks after injection. Kidneys were harvested and processed for histological and ultrastructural analyses after the 16-week follow-up.

Urine and serum analyses. Urinary albumin and urinary or serum creatinine, respectively, were measured using mouse albumin-specific ELISA (Bethyl) and creatinine kits (Labor-Technik). Proteinuria was expressed as $\mathrm{mg}$ albumin $/ \mathrm{mg}$ creatinine. Blood glucose was measured using Accu-Check Sensor (51).

Blood pressure analysis. Blood pressure analysis was performed using a Panlab LE5001 control unit using a single animal heating unit and a LE5160MM transducer and cuff. Animals were accommodated to the equipment for 3 days before measurements were taken. Blood pressure values represent the mean systolic arterial pressure of 3 consecutive measurements for each mouse.

Morphological analysis. Kidneys were fixed in $4 \%$ paraformaldehyde and embedded in paraffin, GMA/MMA, Epon (34), or in Lowicryl K4M resin (Electron Microscopy Sciences) and further processed for PAS staining, transmission electron microscopy, or scanning electron microscopy, respectively. Sclerosis index was done as described by el Nahas et al. (24).

Histological analyses. Quantitative stereological analyses of kidney sections were performed as described previously (34). Briefly, the mean glomerular volume (mean $v_{(G l o m)}$ ) was determined from the mean glomerular profile area (mean $\left.A_{(\text {Glom })}\right)(52)$, which was obtained by measuring 100 systematically sampled glomerular profiles per animal. The physical dissector principle was applied for counting podocytes $\left(\mathrm{Q}^{-}\right)$as described, using semithin sections $(53,54)$. The numerical density of podocytes in glomeruli $\left(N_{V(P / G l o m)}\right)$ was calculated as the quotient of the sum of $\mathrm{Q}^{-}$divided by the dissector volume. The number of podocytes per glomerulus $\left(N_{(P, G l o m)}\right)$ was calculated multiplying $N_{V(P / G l o m)}$ and mean $v_{(\text {Glom })}$. The volume fraction of podocytes per glomerulus $\left(V v_{(P / G l o m)}\right)$ was determined by point counting method. The mean podocyte volume (mean $\left.v_{(P)}\right)$ was calculated dividing $V v_{(P / \text { Glom })}$ by $N_{V(P / \text { Glom })}$. The mesangial and capillary volume fractions $\left(V v_{(\text {Mes/Glom })}\right.$ and $V v_{(\text {Cap } / \text { Glom })}$, respectively) were determined on PAS-stained plastic sections by point counting, using a computer-assisted stereology system (NewCast; Visiopharm). Sixteen to 30 whole glomerular profiles were evaluated (corresponding to 205-471 reference points). The total volume of mesangium or capillaries per glomerulus $\left(V_{(\text {Mes, Glom) }}\right.$ and $V_{(\text {Cap, Glom) }}$, respectively) was obtained by multiplying the respective volume fraction by mean $v_{\text {(Glom) }}$. All results were corrected for embedding shrinkage (34).

Immunofluorescence staining of kidney sections. Kidneys were frozen in OCT compound and sectioned at $6 \mu \mathrm{m}$ (Leica Kryostat). The sections were fixed with $4 \%$ paraformaldehyde, blocked in PBS containing $5 \%$ BSA, and incubated for 1 hour with primary antibodies as indicated. After PBS rinse for several times, fluorophore-conjugated secondary antibodies (Invitrogen) were applied for 30 minutes. Images were taken using a Zeiss laser scan microscope equipped with a $\times 63$ water immersion objective or a Zeiss fluorescence microscope equipped with $\times 20$ and $\times 40$ oil immersion objectives.

Quantification of immunofluorescence results. Images were acquired by a Zeiss Axioscope 40FL microscope, equipped with AxioCam MRc5 digital video camera and immunofluorescence apparatus (Carl Zeiss SpA). Images were recorded using AxioVision software 4.3 and analyzed by the AxioVision analysis module (Carl Zeiss SpA). Glomeruli were selected as region of interest (ROI), and a macro (consisting of a color threshold procedure, followed by filtering and Danielsson's algorithm) was applied to select stained areas and to calculate their relative quantity as a percentage of the ROI area.

Isolation and characterization of adult mouse glomeruli. Glomeruli were isolated using Dynabead perfusion and were glass-glass-homogenized in lysis buffer (containing 20 mM CHAPS and 1\% Triton X-100) (55). After centrifugation $\left(15,000 \mathrm{~g}, 15\right.$ minutes, $\left.4^{\circ} \mathrm{C}\right)$ protein concentration was determined by Dc Protein-Assay (Bio-Rad). Equal amounts of protein were separated on SDS page. Western blots were densitometrically analyzed using LabImage software. Ratios of protein band intensity to loading control protein band intensity are shown (Figures 1C, 3C, 6F, and 7C).

Patients and microarray analysis. Human renal biopsies from patients and controls were collected with informed consent within the framework of the European Renal cDNA Bank - Kröner-Fresenius Biopsy Bank (35) and the Pima Indian Diabetes Study (56), respectively. The former biopsy bank has been approved by the cantonal ethics committee of Zurich, Switzerland, the latter by the National Institute of Diabetes and Digestive and Kidney Diseases in Bethesda, Maryland, USA. The approval allows exclusively the report of aggregate data; no individual data are reported or deposited. Glomeruli were microdissected, total RNA isolated, linearly amplified, and hybridized to Affymetrix HG-U 133 Plus 2.0 microarrays as reported previously (57). A single probe-based analysis tool, ChipInspector (Genomatix Software $\mathrm{GmbH}$ ) was used for transcript annotation, total intensity normalization, significance analysis of microarrays, and transcript identification based on significantly changed probe signals (58). Only probes that match uniquely to the genome and to at least 1 transcript (or overlapping transcripts) were retained for further analysis. The input data for the SAM analysis were single probe values, and the resulting probes with significant alteration of signal levels were subsequently matched with the corresponding transcripts (which may be more than 1 per gene). No fold change cut-off was applied, but minimum coverage of probes with significant alteration per transcripts was set as 3 . This permitted the detection of transcript alterations even below conventional fold change cut-off levels. Shown are the fold changes of the transcripts with the highest probe coverage of each gene.

Antibodies. Antibodies were obtained from Cell Signaling (anti-mTOR rabbit mAb, 2983; anti-Raptor rabbit mAb, 2280; anti-Rictor rabbit mAb, 2114; anti-Rictor rabbit mAb, 9476; anti-pS6 rabbit mAb, 4857; anti-pS6 rabbit mAb, 4858; anti-S6 rabbit mAb, 2217; anti-pAKT T308 rabbit mAb, 2965; anti-AKT rabbit pAb, 9272), Millipore (anti-pPKCE S729 rabbit pAb, 06-821; anti-Nidogen rat mAb, MAB1946; anti-Par3 rabbit pAb, 07-330), Progen (anti-Nephrin guinea pig pAb, GP-N2; anti-synaptopodin mouse mAb, G1D4, 65194), Sigma-Aldrich (anti- $\alpha$-tubulin mouse mAb, T6199; anti- $\beta$-actin mouse mAb, A1978; anti-podocin rabbit pAb, P0372), and AbD Serotec (anti-CD68 rat mAb, FA-11, MCA 1957T). Nuclear staining reagents and fluorophore-conjugated secondary antibodies were obtained from Invitrogen (To-Pro-3, T3605; Alexa Fluor 488 goat anti-guinea pig IgG, A11073; Alexa Fluor 555 goat anti-guinea pig IgG, A21435; Alexa Fluor 555 goat anti-rat IgG, A21434; Alexa Fluor 488 goat anti-rat IgG, A11006; Alexa Fluor 488 donkey anti-rabbit, A21206).

Statistics. Data are expressed as the mean \pm SEM. All shown data reflect a minimum of 3 subjects per test. Statistical comparisons were performed using 2-tailed Student's $t$ test if not stated otherwise. Differences with $P<0.05$ were considered significant.

\section{Acknowledgments}

We thank Dörte Thiel, Charlotte Meyer, (Renal Division, University Hospital Freiburg), Elisabeth Wieser, Brigitte Langer (Pathology, Medical University Vienna), Angela Siebert, Heike Sperling (Veterinary Pathology, Munich), and Evelyn Wätzig (Pathology, University Hospital Freiburg) for excellent technical assistance. We thank Michael Köttgen and Sebastian Arnold for carefully reading the manuscript. We thank all participating centers of the European Renal cDNA Bank - Kröner-Fresenius biopsy bank and their patients for their cooperation. Active members at the time of the study are listed in ref. 58 and at http://www.forschungsportal. ch/unizh/p9291.htm. This study was supported by the Intramural Research Program of the National Institute of Diabetes and Digestive and Kidney Diseases (NIDDK) (to R.G. Nelson; NIDDK P30 
DK081943 to M. Kretzler), the Swiss National Science Foundation (32-122439/1 to C.D. Cohen) and the Else Kröner-Fresenius-Stiftung (A62/04 to C.D. Cohen), the NIH (DK083491 to K. Inoki), Deutsche Forschungsgemeinschaft (DFG) grants (HU 1016/2-1, SFB 592 B10, and P7-KFO 201 to T.B. Huber), the Excellence Initiative of the German Federal and State Governments (EXC 294), and BMBF Gerontosys-Project NephAge.
Received for publication August 16, 2010, and accepted in revised form March 8, 2011.

Address correspondence to: Tobias B. Huber, Renal Division, University Hospital Freiburg, Breisacher Str. 66, 79106 Freiburg, Germany. Phone: 49.761.270.3559; Fax: 49.761.270.3270; E-mail: tobias.huber@uniklinik-freiburg.de.
1. Estacio RO, Schrier RW. Diabetic nephropathy: pathogenesis, diagnosis, and prevention of progression. Adv Intern Med. 2001;46:359-408.

2. Wullschleger S, Loewith R, Hall MN. TOR signaling in growth and metabolism. Cell. 2006;124(3):471-484.

3. Holz MK, Ballif BA, Gygi SP, Blenis J. mTOR and S6K1 mediate assembly of the translation preinitiation complex through dynamic protein interchange and ordered phosphorylation events. Cell. 2005;123(4):569-580.

4. Shahbazian D, et al. The mTOR/PI3K and MAPK pathways converge on eIF4B to control its phosphorylation and activity. EMBOJ. 2006;25(12):2781-2791.

5. Montagne J, Stewart MJ, Stocker H, Hafen E, Kozma SC, Thomas G. Drosophila S6 kinase: a regulator of cell size. Science. 1999;285(5436):2126-2129.

6. Zhang H, Stallock JP, Ng JC, Reinhard C, Neufeld TP. Regulation of cellular growth by the Drosophila target of rapamycin dTOR. Genes Dev. 2000; 14(21):2712-2724.

7. Laplante M, Sabatini DM. mTOR signaling at a glance. J Cell Sci. 2009;122(pt 20):3589-3594.

8. Polak P, Hall MN. mTOR and the control of whole body metabolism. Curr Opin Cell Biol. 2009; 21(2):209-218

9. Torras J, et al. Rapamycin has dual opposing effects on proteinuric experimental nephropathies: is it a matter of podocyte damage? Nephrol Dial Transplant. 2009;24(12):3632-3640.

10. Mori H, et al. The mTOR pathway is highly activated in diabetic nephropathy and rapamycin has a strong therapeutic potential. Biochem Biophys Res Commun. 2009;384(4):471-475.

11. Inoki K. Role of TSC-mTOR pathway in diabetic nephropathy. Diabetes Res Clin Pract. 2008; 82 suppl 1:S59-S62.

12. Lloberas $\mathrm{N}$, et al. Mammalian target of rapamycin pathway blockade slows progression of diabetic kidney disease in rats. J Am Soc Nephrol. 2006;17(5):1395-1404.

13. Sakaguchi M, Isono M, Isshiki K, Sugimoto T, Koya $\mathrm{D}$, Kashiwagi A. Inhibition of mTOR signaling with rapamycin attenuates renal hypertrophy in the early diabetic mice. Biochem Biophys Res Commun. 2006;340(1):296-301.

14. Rangan GK, Coombes JD. Renoprotective effects of sirolimus in non-immune initiated focal segmental glomerulosclerosis. Nephrol Dial Transplant. 2007;22(8):2175-2182.

15. Amer H, Cosio FG. Significance and management of proteinuria in kidney transplant recipients. $J \mathrm{Am}$ Soc Nephrol. 2009;20(12):2490-2492.

16. Munivenkatappa R, Haririan A, Papadimitriou JC, Drachenberg CB, Dinits-Pensy M, Klassen DK. Tubular epithelial cell and podocyte apoptosis with de novo sirolimus based immunosuppression in renal allograft recipients with DGF. Histol Histopathol. 2010;25(2):189-196.

17. Letavernier E, et al. High sirolimus levels may induce focal segmental glomerulosclerosis de novo. Clin J Am Soc Nephrol. 2007;2(2):326-333.

18. Sarbassov DD, et al. Prolonged rapamycin treatment inhibits mTORC2 assembly and Akt/PKB. Mol Cell. 2006;22(2):159-168.

19. Moeller MJ, Sanden SK, Soofi A, Wiggins RC, Holzman LB. Podocyte-specific expression of cre recombinase in transgenic mice. Genesis. 2003;35(1):39-42.

20. Bentzinger CF, et al. Skeletal muscle-specific abla- tion of raptor, but not of rictor, causes metabolic changes and results in muscle dystrophy. Cell Metab. 2008;8(5):411-424.

21. Polak P, Cybulski N, Feige JN, Auwerx J, Ruegg MA, Hall MN. Adipose-specific knockout of raptor results in lean mice with enhanced mitochondrial respiration. Cell Metab. 2008;8(5):399-410.

22. $\mathrm{Um} \mathrm{SH}$, et al. Absence of S6K1 protects against ageand diet-induced obesity while enhancing insulin sensitivity. Nature. 2004;431(7005):200-205.

23. Harrington LS, et al. The TSC1-2 tumor suppressor controls insulin-PI3K signaling via regulation of IRS proteins. J Cell Biol. 2004;166(2):213-223.

24. el Nahas AM, Bassett AH, Cope GH, Le Carpentier JE. Role of growth hormone in the development of experimental renal scarring. Kidney Int. 1991;40(1):29-34.

25. GangloffYG, et al. Disruption of the mouse mTOR gene leads to early postimplantation lethality and prohibits embryonic stem cell development. Mol Cell Biol. 2004;24(21):9508-9516.

26. Murakami M, et al. mTOR is essential for growth and proliferation in early mouse embryos and embryonic stem cells. Mol Cell Biol. 2004;24(15):6710-6718.

27. Eremina V, et al. VEGF inhibition and renal thrombotic microangiopathy. N Engl J Med. 2008; 358(11):1129-1136.

28. Muzumdar MD, Tasic B, Miyamichi K, Li L, Luo L. A global double-fluorescent Cre reporter mouse. Genesis. 2007;45(9):593-605

29. Jones N, et al. Nck proteins maintain the adult glomerular filtration barrier. J Am Soc Nephrol. 2009; 20(7):1533-1543

30. Sarbassov DD, Guertin DA, Ali SM, Sabatini DM. Phosphorylation and regulation of Akt/ PKB by the rictor-mTOR complex. Science. 2005; 307(5712):1098-1101.

31. Weening JJ, Van Guldener C, Daha MR, Klar N, van der Wal A, Prins FA. The pathophysiology of protein-overload proteinuria. Am J Pathol. 1987; 129(1):64-73.

32. Morita $\mathrm{H}$, et al. Heparan sulfate of perlecan is involved in glomerular filtration. J Am Soc Nephrol. 2005;16(6):1703-1710.

33. Osterby R, Gundersen HJ. Glomerular size and structure in diabetes mellitus. I. Early abnormalities. Diabetologia. 1975;11(3):225-229.

34. Herbach N, et al. Diabetic kidney lesions of GIPRdn transgenic mice: podocyte hypertrophy and thickening of the GBM precede glomerular hypertrophy and glomerulosclerosis. Am J Physiol Renal Physiol. 2009;296(4):F819-F829.

35. Cohen CD, Frach K, Schlondorff D, Kretzler M. Quantitative gene expression analysis in renal biopsies: a novel protocol for a high-throughput multicenter application. Kidney Int. 2002;61(1):133-140.

36. Inoki $\mathrm{K}$, etal. mTORC1 activation in podocytes is a critical step in the development of diabetic nephropathy in mice. J Clin Invest. 2011;121(6):2181-2196.

37. Welsh GI, et al. Insulin signaling to the glomerular podocyte is critical for normal kidney function. Cell Metab. 2010;12(4):329-340.

38. Skov K, Nyengaard JR, Patwardan A, Mulvany MJ. Large juxtamedullary glomeruli and afferent arterioles in healthy primates. Kidney Int. 1999; 55(4):1462-1469.

39. Artacho-Perula E, Roldan-Villalobos R, Salcedo-Leal I, Vaamonde-Lemos R. Stereological estimates of volume-weighted mean glomerular volume in strepto- zotocin-diabetic rats. Lab Invest. 1993;68(1):56-61.

40. Souster LP, Emery JL. The sizes of renal glomeruli in fetuses and infants. J Anat. 1980;130(pt 3):595-602.

41. Samuel T, Hoy WE, Douglas-Denton R, Hughson MD, Bertram JF. Determinants of glomerular volume in different cortical zones of the human kidney. J Am Soc Nephrol. 2005;16(10):3102-3109.

42. Lieberthal W, Levine JS. The role of the mammalian target of rapamycin (mTOR) in renal disease. $J \mathrm{Am}$ Soc Nephrol. 2009;20(12):2493-2502.

43. Wiggins JE, et al. Podocyte hypertrophy, "adaptation," and "decompensation" associated with glomerular enlargement and glomerulosclerosis in the aging rat: prevention by calorie restriction. J Am Soc Nephrol. 2005;16(10):2953-2966.

44. Kretzler M. Role of podocytes in focal sclerosis: defining the point of no return. J Am Soc Nephrol. 2005;16(10):2830-2832.

45. Niranjan T, et al. The Notch pathway in podocytes plays a role in the development of glomerular disease. Nat Med. 2008;14(3):290-298.

46. Bielesz B, et al. Epithelial Notch signaling regulates interstitial fibrosis development in the kidneys of mice and humans. J Clin Invest. 2010; 120(11):4040-4054.

47. Sharma S, Sirin Y, Susztak K. The story of Notch and chronic kidney disease. Curr Opin Nephrol Hypertens. 2011;20(1):56-61

48. Hartleben B, et al. Autophagy influences glomerular disease susceptibility and maintains podocyte homeostasis in aging mice. J Clin Invest. 2010;120(4):1084-1096.

49. Choi YJ, et al. Tuberous sclerosis complex proteins control axon formation. Genes Dev. 2008; 22(18):2485-2495.

50. Meier M, et al. Deletion of protein kinase C-beta isoform in vivo reduces renal hypertrophy but not albuminuria in the streptozotocin-induced diabetic mouse model. Diabetes. 2007;56(2):346-354.

51. Izzedine H, Brocheriou I, Frances C. Post-transplantation proteinuria and sirolimus. NEngl J Med. 2005;353(19):2088-2089.

52. Weibel ER, Gomez DM. A principle for counting tissue structures on random sections. J Appl Physiol. 1962;17:343-348.

53. Hoeflich A, et al. Insulin-like growth factor binding protein 2 (IGFBP-2) separates hypertrophic and hyperplastic effects of growth hormone $(\mathrm{GH}) / \mathrm{IGF}-\mathrm{I}$ excess on adrenocortical cells in vivo. FASEB J. 2002;16(13):1721-1731.

54. Sterio DC. The unbiased estimation of number and sizes of arbitrary particles using the disector. J Microsc. 1984;134(pt 2):127-136.

55. Takemoto $\mathrm{M}$, et al. A new method for large scale isolation of kidney glomeruli from mice. Am J Pathol. 2002;161(3):799-805.

56. Berthier CC, et al. Enhanced expression of Janus kinase-signal transducer and activator of transcription pathway members in human diabetic nephropathy. Diabetes. 2009;58(2):469-477.

57. Cohen $\mathrm{CD}$, et al. Comparative promoter analysis allows de novo identification of specialized cell junction-associated proteins. Proc Natl Acad Sci US A. 2006;103(15):5682-5687.

58. Cohen CD, et al. Improved elucidation of biological processes linked to diabetic nephropathy by single probe-based microarray data analysis. PLOS One. 2008;3(8):e2937. 